\title{
Consistency in Nervous Systems: Trial-to-Trial and Animal-to-Animal Variations in the Responses to Repeated Applications of a Sensory Stimulus in Aplysia
}

\author{
Jian-young Wu, ${ }^{1,3, a}$ Yang Tsau, ${ }^{1,3}$ Hans-Peter Hopp, ${ }^{1,3, b}$ Lawrence B. Cohen, ${ }^{1,3}$ Akaysha C. Tang, ${ }^{1,2}$ \\ and Chun Xiao Falk ${ }^{1,3}$ \\ 'Department of Physiology, Yale University School of Medicine, New Haven, Connecticut 06510, ${ }^{2}$ Department of \\ Psychology, Harvard University, Cambridge, Massachusetts 02138, and ${ }^{3}$ Marine Biological Laboratory, Woods Hole, \\ Massachusetts 02543
}

What is the internal noise in a nervous system? We studied this question by determining the trial-to-trial consistency of the neuronal response in the abdominal ganglion of Aplysia californica. Because our voltage-sensitive dye recordings detected the spike activity from a large fraction of the neurons in the ganglion, these results provide a reasonably complete characterization of the consistency of the response to a sensory stimulus. The consistency of each neuron was evaluated by the number and timing of spikes in the response.

The variability in the spike count was described using the coefficient of variation. The spike count variations follow a Poisson distribution, indicating that most of these variations were the result of a random process. For each neuron the reliability of the response to touch was measured in two ways; both measures indicated a broad distribution of reliabilities within the neuron population.

The time of the maximum response also varied substantially in some animals. These timing variations were in part due to random processes and in part due to systematic effects (changes in activity of many neurons that were highly correlated).

The time course of the activity of individual neurons was compared with the time course of the gill withdrawal. In some animals the activity of individual neurons was only poorly correlated with the behavior; in contrast, the summed activity of groups of neurons matched the behavior quite well. This implies that the behavioral output of the system may be a distributed combination of the activity of many neurons.

The differences between animals were substantially larger

Received Jan. 25, 1993; revised May 10, 1993; accepted Aug. 12, 1993

We are grateful to Ray Falk, Markus Meister, Barak Pearlmutter, Brian Salzberg, Sebastian Seung, and Hiam Sompolinsky for advice about the statistical measures. We also thank the journal reviewers for helpful suggestions concerning the manuscript. We are grateful to David Senseman for the loan of the 464 element photodiode array and the Silicon Graphics workstation. Vic Pantani and Henrik Abildgaard of the Physiology electronics shop designed and constructed the amplifiers and analog-to-digital converter used to record the output of the diode array. This work was supported in part by NINDS Grant NS08437, an IBM and a William James Fellowship, and NIH National Research Service Award NS07102.

Correspondence should be addressed to Lawrence B. Cohen, Department of Cellular and Molecular Physiology, Yale University School of Medicine, 333 Cedar Street, New Haven, CT 06510.

'Present address: Department of Zoology, University of Maryland, College Park, MD, 20742.

'Present address: Max-Planck Institut fur Entwicklungsbiologie, D7400 Tubingen, Gennany.

Copyright $(C 1994$ Society for Neuroscience $0270-6474 / 94 / 141366-19 \$ 05.00 / 0$ than the trial-to-trial differences in one animal. The responses made by different preparations differed along many dimensions.

IKey words: consistency, voltage-sensitive dye, optical recording, Aplysia, trial-to-trial differences, animal-to-animal differences]

The nervous system can produce reasonably consistent behavioral outputs when presented with identical input stimuli. This implies that identical representations of behavioral output must exist at some level, even if only in the muscular effectors themselves. At one extreme of possible functional architectures, only the activity of the muscles would be identical across trials. All the neurons between the sensory input and the muscles could be active in varying combinations as long as these combinations cause the same activation pattern in the muscles. At the other extreme, all the individual cells that participate in a behavior would function identically from one trial to the next. One way to discriminate between these possible functional architectures is to determine whether the activity of individual neurons has the required consistency or whether it is necessary to use the summed activity of groups of neurons to obtain this consistency.

Early results had suggested a very simple functional architecture for the gill withdrawal reflex. Byrne et al. (1978) reported that $60 \%$ of the Aplysia gill-withdrawal could be accounted for by the monosynaptic connection between LE sensory neurons and gill motor neurons. However, more recent evidence from microclectrode studics has substantially lowered the contribution of the monosynaptic component and demonstrated the contribution of other, as yet unidentified, sensory neurons as well as contributions from several tens of interneurons (Hawkins et al., 1981; Frost et al., 1988; Cohen et al., 1991; Trudeau and Castellucci, 1992). Furthermore, optical measurements demonstrated that the number of neurons activated by the siphon stimulation is approximately 300 (Zecevic et al., 1989; Nakashima et al., 1992). Falk et al. (1993), applying cluster analysis to spike data obtained from habituation experiments, identified several groups of neurons that exhibited common characteristics along two temporal dimensions. Just like single cells, such groups of functionally similar cells might represent functional units themselves. Taken together, the above results clearly suggest a much more complex functional architecture for the abdominal ganglion.

Other investigators have studied trial-to-trial and animal-toanimal variability at several organizational levels: (1) at indi- 
vidual synapses, (2) in counts of the number of neurons, and (3) in spike counts in the responses of individual neurons. Gardner (1986) has studied the variability in synaptic currents between identified pairs of neurons in the Aplysia buccal ganglion. In one preparation he measured the postsynaptic response to a series of 136 action potentials elicited at $10 \mathrm{sec}$ intervals. The postsynaptic conductances averaged $0.5 \mu \mathrm{S}$ and ranged from 0.3 to $0.8 \mu \mathrm{S}$. Gardner also examined the same synapse in $31 \mathrm{dif}-$ ferent animals. In contrast, the mean synaptic conductances ranged from 0.028 to $1.7 \mu \mathrm{S}$ in the 31 animals. This 60 -fold difference in synaptic strength among animals is much larger than the trial-to-trial range in one animal; the animal-to-animal differences are striking.

Looking at a second measure of animal-to-animal variability, London et al. (1987) counted the number of neurons present in the buccal ganglia of Navanax inermis and reported a mean of 200 with an SD of 40 . There were substantial animal-to-animal differences in the number of neurons in these opisthobranch ganglia. From these measurements of synaptic strength and numbers of neurons, we anticipated that the response to touch in Aplysia would show greater animal-to-animal than trial-totrial differences.

The trial-to-trial variations in the spike response of individual cells have been studied in cortical neurons over the past several decades (e.g., Kuffler et al., 1957; Werner and Mountcastle, 1963; Heggelund and Albus, 1978; Tolhurst et al., 1983). The variability in spike counts, as expressed by the coefficient of variation (SD normalized for the mean response), always decreased as the magnitude of the response increased. For some data sets (Heggelund and Albus, 1978; Tolhurst et al., 1983) the coefficient of variation was inversely proportional to the square root of the mean response. This is the expected relationship if the distribution of responses is Poisson.

We have made similar measurements of variability in the spike response of individual Aplysia neurons but have simultaneously recorded the activity of up to half of the neurons in the abdominal ganglion and thus obtained a relatively complete picture of the variability. Monitoring the activity of many neurons allowed us to determine whether trial-to-trial differences are apparently random or are systematic. By systematic differences we mean trial-to-trial changes where the activities of many neurons change in a correlated fashion. Furthermore, because we obtain a relatively complete sample of neuron activity from each animal, we can also compare responses between animals.

The voltage-sensitive dye signal from some neurons in the Aplysia abdominal ganglion is smaller than the noise. The most complete recordings with a 124 pixel photodiode array (used in initial experiments) monitored the activity of about $30 \%$ of the neurons (Zecevic et al., 1989); the best recordings made with a 464 pixel array (used in later experiments) are about $50 \%$ complete (see Materials and Methods).

Our initial experiments studying trial-to-trial consistency $(\mathrm{Wu}$ et al., 1989; Tang et al., 1992) were carried out using a mechanical touch as a stimulus. To rulc out the possibility that differences in the response were simply due to differences in the stimuli, we have employed two additional types of stimulation: a suction electrode stimulus to the siphon skin and an intracellular microelectrode stimulation of a single neuron in the ganglion.

The responses of the individual neurons across trials are given by the number and timing of action potentials that follow the stimulus. In order to describe the trial-to-trial variability in a relatively quantitative way, we evaluated four important and characteristic aspects of the response of individual neurons across trials. The first two were based on the number of spikes (but ignored information about timing); the last two were based on timing of the spikes (but ignored information about the number).

(1) The first measure was a simple characterization of the trial-to-trial variability in the number of spikes (spike counts) in the poststimulus period using the coefficient of variation (the standard deviation divided by the mean).

(2) Second, we estimated the reliability of the response of individual neurons: how reliably each neuron functioned as a detector of the siphon stimulus. Here we compared the spike counts in the pre- and poststimulus periods. The probability of correct discrimination between the stimulus and no stimulus was used to determine how often the poststimulus spike count could be confused with a prestimulus count. Because the decision criterion for the probability of correct discrimination is determined from the mean responses a posteriori, this measure does not address an issue that the animal faces, that of deciding in each trial whether a stimulus occurred. We also calculated a response probability using an a priori criterion for determining whether a neuron had responded. Each neuron was scored as responding or not responding in each trial and the response probability score was simply the fraction of the trials in which the neuron responded.

(3) To obtain a measure of the trial-to-trial variations in the timing of spike activity, we determined the time of maximum spike rate for each neuron in each trial. The time of maximum rate in individual trials was compared with the mean of the peak times for that neuron.

(4) Fourth, we compared the timing of activity of cach individual neuron with the timing of the behavioral output of the system (the gill-withdrawal).

\section{Materials and Methods}

The trial-to-trial consistency results come from eight preparations: three using a mechanical touch to the siphon, four using a suction electrode stimulation of the siphon, and one using a microelectrode stimulation of an individual neuron in the abdominal ganglion. An additional 26 animals that received a mechanical stimulus are included in the animalto-animal comparisons. The animals weighed between 5 and $20 \mathrm{gm}$ The experiments were carried out on the isolated siphon preparation developed by Kupfermann et al. (1971) (Fig. 1). The siphon was connected to the abdominal ganglion via the siphon nerve and the ganglion communicated with the gill via the branchial and genital nerves. The absence of a direct peripheral connection between the siphon and gill means that the gill withdrawal must be mediated via the abdominal ganglion. The ganglia were not desheathed. In later experiments the gillwithdrawal was recorded on videotape. The chamber temperature was kept at $15 \pm 1{ }^{\circ} \mathrm{C}$. Additional details concerning the preparation are given in Falk et al. (1993).

\section{Stimulation}

For the experiments using the mechanical touch and suction electrode stimuli we bathed the siphon in synaptic blocker solution containing $220 \mathrm{mM} \mathrm{MgCl}_{2}, 1 \mathrm{mM} \mathrm{CaCl}_{2}, 228 \mathrm{~mm} \mathrm{NaCl}, 10 \mathrm{~mm} \mathrm{KCl}$, and $10 \mathrm{~mm}$ Tris- $\mathrm{HCl}$. This solution was used to block siphon movement to achieve a consistent stimulation site. Control experiments indicated that synaptic blocker in the siphon chamber had no detectable effect on the touch response of neurons in the ganglion or on the gill-withdrawal reflex.

Mechanical toucher. Mechanical stimulation was delivered to the siphon skin via a computer-controlled motor that moved a $0.6 \mathrm{~mm}$ glass rod with a rounded tip. To reduce possible effects of variation in stimulus strength we used a stimulus that was not on a steep part of the stimulusresponse curve. In four experiments we found that the mean extrapolated stimulus threshold for the gill-withdrawal reflex was $0.3 \pm 0.2$ 
Figure 1. Schematic drawing of the preparation and apparatus. Light from a tungsten halogen lamp was passed through a $720 \pm 25 \mathrm{~nm}$ interference filter and focused on the preparation using a modification of Kohler illumination. The condenser iris was opened so that the condenser numerical aperture would equal the objective numerical aperture. A $12 \times 12$ or a 464 element photodiode array was placed at the plane where the objective forms the real, inverted image. The photodiode array records the light transmitted by the ganglion. The outputs from each diode were individually amplified. The signals were digitized by a dual analogto-digital converter and stored in a Motorola 68000 VME bus computer. The inset at the lower right shows schematically the relative positions of the image of the ganglion and the 464 element photodiode array.

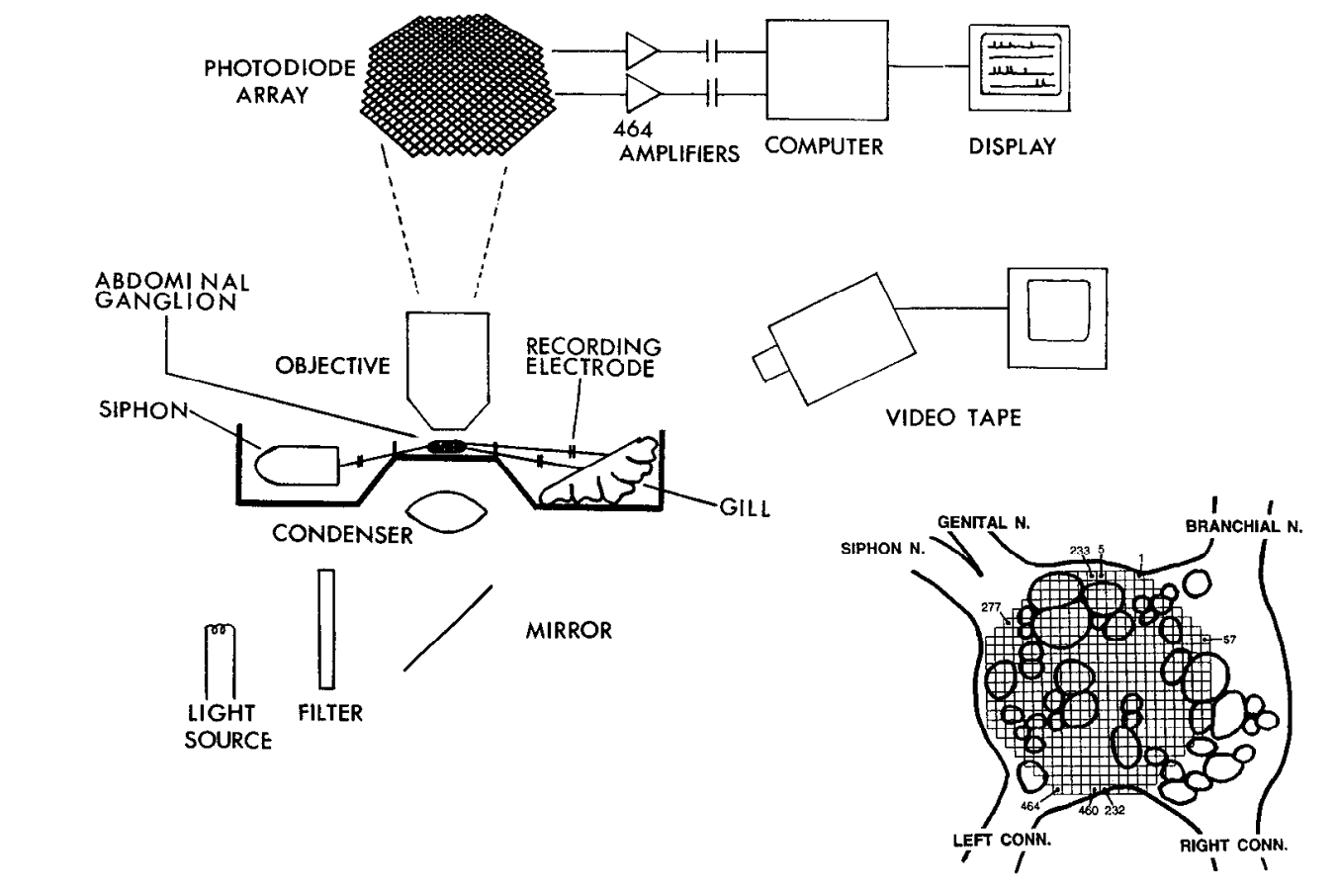

gm. In all four preparations, a $1.0 \mathrm{gm}$ increase in stimulus strength was enough to get past the steep part of the stimulus-response curve. For the measurements of trial-to-trial variability in this article, we began with a test stimulus of $1.0 \mathrm{gm}$. Preparations with no gill-withdrawal in response to the test stimulus were discarded. A stimulus strength of 2.0 gm was used in the experiments.

Suction electrode. The suction electrode was made from a polished glass tube with an outer diameter of $600 \mu \mathrm{m}$ and an inner diameter of $200 \mu \mathrm{mI}$. Silver-silver chloride wires were used both internally and externally. The suction electrode was positioned on the siphon skin and then gentle suction was applied and maintained for the duration of the experiment.

Intracellular electrode. We impaled a large neuron in the upper left quadrant, medial and posterior to the exit point of the siphon nerve. From its size, location, and the existence of irregular inhibitory synaptic potentials, we think that this cell may be L10 (Koester and Kandel, 1977). Microelectrodes of 20-30 M resistance were pulled using a Sutter Instrument model P87 puller. To facilitate impalement the ganglia were incubated in a $0.2 \%$ trypsin (Sigma) solution for 5-15 min followed by a $0.25 \%$ solution of trypsin inhibitor (Sigma) for $10 \mathrm{~min}$.

\section{Optical recording}

We used two voltage-sensitive pyrazolone-oxonol dyes: NK3041 (Grinvald et al., 1980; obtained from Nippon Kankoh Shikiso Kenkyusho Co. Ltd., Okayama, Japan) and its diethyl analog, JPW 1131 (obtained from Dr. Leslie Loew, Department of Physiology, University of Connecticut, Farmington, CT). These dyes were first suggested and synthesized by Rina Hildesheim and Amiram Grinvald as RH 155 and RH479. The ganglia were stained with a dye concentration of $0.15 \mathrm{mg} / \mathrm{ml} \mathrm{fol-}$ lowing the staining protocol of Nakashima et al. (1992). Photostimulation, activation of neuron activity by the measuring light, was seen in a few preparations using NK3041. These effects did not seem to be present with JPW 1131. The largest number of trials we recorded was 10 . In the best preparations the measurements would be limited to 1530 trials by the reduction in signal size that occurs as a result of dye bleaching. The oxonol dyes mainly stained the cell body layer; the neuropil region was only weakly stained. As a result, optical signals from neuropil processes or axons were rarely detected.

The initial measurements were made using 124 elements of a $12 \times$ 12 array (Centronic Inc., Newbury Park, CA). Later experiments were carried out with a 464 element photodiode array (in a $24 \times 24$ grid with the corner elements omitted) using methods that were modifications of those previously published (Salzberg et al., 1977; Grinvald et al., 1981; London et al., 1987; Zecevic et al., 1989, Wu and Cohen, 1993). For the recordings with both arrays we sampled 8192 data points per detector per trial. With the 124 element array the duration of the recording was $8 \mathrm{sec}(1.0 \mathrm{msec}$ per data point). Because of hardware limitations, with the 464 element array the duration of the fastest 8192 data point recording we could make was $13 \mathrm{sec}(1.6 \mathrm{mscc}$ per data point).

The best (most complete) recordings from Aplysia with the 124 pixel array were estimated to detect the activity of about $30 \%$ of the neurons in the abdominal ganglion (Zecevic et al., 1989). Nakashima et al. (1992) found that 2.5 times as many cells were detected with a 448 element array than with a 100 element array. In their comparison different preparations were used with the two arrays. We analyzed one trial from the 464 pixel array and compared that result with the analysis of the same data binned into a 124 pixel array and found that using the larger array increased the number of detected cells by a factor of 1.5 . In the absence of a direct measurement, we estimate that the most successful recordings made with the 464 pixel array are about $50 \%$ complete.

The diode array was placed in the image plane formed by a Leitz $25 \times 0.4$ NA objective (Fig. 1). The ganglion was positioncd with its ventral side facing the objective and with the corner where the siphon nerve exits the ganglion positioned at the upper left edge of the field of view (Fig. 1, lower right). With this magnification, about $25 \%$ of the image of the ganglion will fall outside the active area of the photodiode array, and active neurons in this region will be missed. This is another factor contributing to the incompleteness of the optical recording from the Aplysia ganglion.

The raw data from the optical recordings consist of cell-body spike signals on photodiode outputs; further analysis is required to determine the activity of individual neurons from this data (for examples and additional details of the sorting methods, see London et al., 1987; Zecevic et al., 1989; Falk et al., 1993). To analyze experiments with several recordings from one preparation, we first concatenated the raw data from all trials so that the analysis could be done as if it were one long recording. In this way we could follow the activity of individual neurons throughout all of the trials of one experiment. Because of variability in the number and position of neurons from ganglion to ganglion we were not able to follow neurons across animals.

The numbering scheme used for the photodiodes in the 464 element array is indicated on the schematic drawing of the array in the lower right of Figure 1. To obtain the identification numbers given on the left of Figures 2, 3, 7, and 10, we began with the detector number that was used for recording the spike times of each neuron; usually this detector had the largest signal from that neuron. If a detector was used for 
recording the activity of more than one neuron, we added 1000 to the ID number for each reuse.

\section{Statistical calculations}

Coefficient of variation. The mean response (mean spike count in a poststimulus interval) across trials was determined for each neuron. We then calculated the standard deviation and the coefficient of variation (the standard deviation normalized for the mean) of the number of spikes for each neuron. We also calculated the theoretical relationship between coefficient of variation and mean response using the assumption that the distribution was Poisson; the standard deviation for a Poisson distribution is the square root of the mean. The calculated values were used to plot the curves in Figures 4 and 9.

Probability of correct discrimination. (Green and Swets, 1966). The probability of correct discrimination between the stimulus and no stimulus was used to determine how often the poststimulus spike count could be confused with a prestimulus count. This calculation used an optimized decision criterion that yields the maximum fraction of correct decisions. The probability of correct discrimination is inversely proportional to the overlap in the distribution of spike counts in the preand poststimulus intervals. Smaller overlaps result from larger differences in the mean pre- and poststimulus spike counts and yield larger probabilities of correct discrimination. The mean response to the stimulus (spike count in the poststimulus 2048 data points) and mean spike count for the prestimulus 2048 data points were determined for each neuron. For the microelectrode experiment (see Fig. 5, bottom) we used the spike count in the 6144 poststimulus data points divided by 3 as the poststimulus mean. We calculated two Poisson distributions of spike counts for each neuron from its pre- and poststimulus means. Equations 1 and 2 were used to calculate the overlap of the two distributions.

$$
P_{\text {overlap }}=P_{\text {post }}(i)+1-P_{\text {pre }}(i)
$$

where $i$ is spike count and

$$
k=\frac{\text { mean(post) }- \text { mean(pre) }}{\ln \left[\frac{\text { mean(post) }}{\text { mean(pre) }}\right]},
$$

where $k$ is the criterion spike count, the count where the two distributions cross. A count larger than $k$ is considered to be a response, and a response smaller than $k$ is considered to be no response. The probability of correct discrimination was determined using

$$
P_{c}=1-P_{\text {overlap }} / 2
$$

The optimum decision criterion for the probability of correct discrimination is determined from the mean responses a posteriori.

Response probability. For the third measure, response probability, we used an a priori criterion for determining whether a neuron had responded. If, for example, the a priori criterion was that there should be less than a 0.05 probability that background activity (determined from the prestimulus spike count) would be erroneously considered a response, the distribution of prestimulus counts was used to estimate the minimum poststimulus spike count that would have occurred by chance with a probability of less than 0.05 . To determine if a neuron made a response to the stimulus in each trial, we had to estimate the probability that a given response might simply result from a chance increase in the background (prestimulus) firing rate. We estimated the expected distribution of background spike counts for each neuron (the number of spikes expected in the poststimulus interval if there was no response to the stimulus) from the number of spikes that occurred in the prestimulus interval. The background spike counts of each neuron were assumed to have a Poisson distribution. The free parameter (rate) of this exponential function was determined by fitting the mean prestimulus spike count across neurons to an exponential curve. The resultant prior distribution gave an excellent fit to the histogram of prestimulus spike counts. This exponential prior distribution of the Poisson rates was then used to calculate the probability of a neuron exhibiting as many poststimulus spikes as were observed, given its prestimulus counts across trials, using Bayes rule (DeGroot, 1986). This calculation was made independently for the poststimulus count of each neuron for each trial. When the chance of a background spike count being as large as the observed poststimulus spike count was less than 0.05 , the neuron was considered to have responded. Each neuron received a binary score (response-no response) for each trial. The response probability of the neuron was the fraction of the trials that were scored as a response.
Time of peak activity. We determined the time of peak activity for each neuron in each trial by determining the minimum interspike interval in the poststimulus period and assigning the peak time to the time of the first spike in the pair with the minimum interval. This procedure required that a cell make two spikes in the poststimulus interval in order to have a peak time determined for that trial. Cells with fewer than three trials with two spikes in the poststimulus interval were removed from the data.

Paired $\mathrm{t}$ test to distinguish systematic differences between trials. The spike counts in the poststimulus 6144 data points were compared pairwise between trials for each neuron. For each pair of trials the mean and SE of the differences between pairs were used to detcrminc the Student's $t$ value and the resulting $p$ value. Because multiple comparisons were made, the $p=0.05$ criterion was adjusted by the number of comparisons using Bonferroni's procedure (Woolson, 1987).

The calculations and graphical outputs for Figures 2-14 were done using MATHEMATICA (Wolfram Research, Champaign, IL; version 2.1) on a Silicon Graphics Iris Indigo workstation.

\section{Results}

\section{Trial-to-trial variations}

We report results from three preparations where a mechanical toucher was used, from four preparations using a suction electrode stimulus to the siphon skin, and from one preparation using an intracellular microelectrode to stimulate an individual neuron in the abdominal ganglion. Figures 2 and 3 illustrate the results from two of these experiments. Each vertical panel represents the results from one trial, each horizontal line represents one neuron, and each vertical tick mark represents one action potential. Figure 2 illustrates the results from one of the experiments using the mechanical toucher, and Figure 3, the results from the experiment with the intracellular stimulus of one neuron. The neurons are arranged from top to bottom in groups using the response probability measure described below. The results from the three types of stimulation are compared in a later section and in Figures 4-6.

Figure 2 illustrates results from all eight recordings in an experiment where the siphon skin received a light touch 3.25 sec after the beginning of each recording (indicated by the dashed lines). For clarity of presentation 62 neurons that made less than 1 spike per trial were omitted from the figure. These neurons are, however, included in the analyses shown in Figures 4-6 and 13. Clearly, there are similarities in the response to touch from one trial to the next. However, a substantial number of the neurons, including many with relatively large responses, show dramatic trial-to-trial differences in the number of action potentials evoked by the stimulus (e.g., cells numbered 292, 77 , $2334,375,3308,2332,1257,1308,2665$, and 368 ). While many of these trial-to-trial differences do not appear to be correlated across neurons and therefore not systematic, systematic changes are also seen in Figure 2. For example, the eighth trial has less activity than the preceding seven trials; there is no delayed component either in the spike density histogram or in the gill withdrawal (shown at the bottom). In addition, there are differences in the timing of the delayed components of the gillwithdrawal in the first seven trials that are reflected in the action potential activity. Thus, in this experiment, as in all those we have examined, there are both apparently random and apparently systematic effects. We first present results and analyses that emphasize the apparently random changes and then present results that emphasize systematic effects.

\section{Variations that are apparently random}

We used the paired $t$ test based on spike counts across successive trials to identify trials with large systematic differences in spike 


\section{MECHANICAL TOUCH}

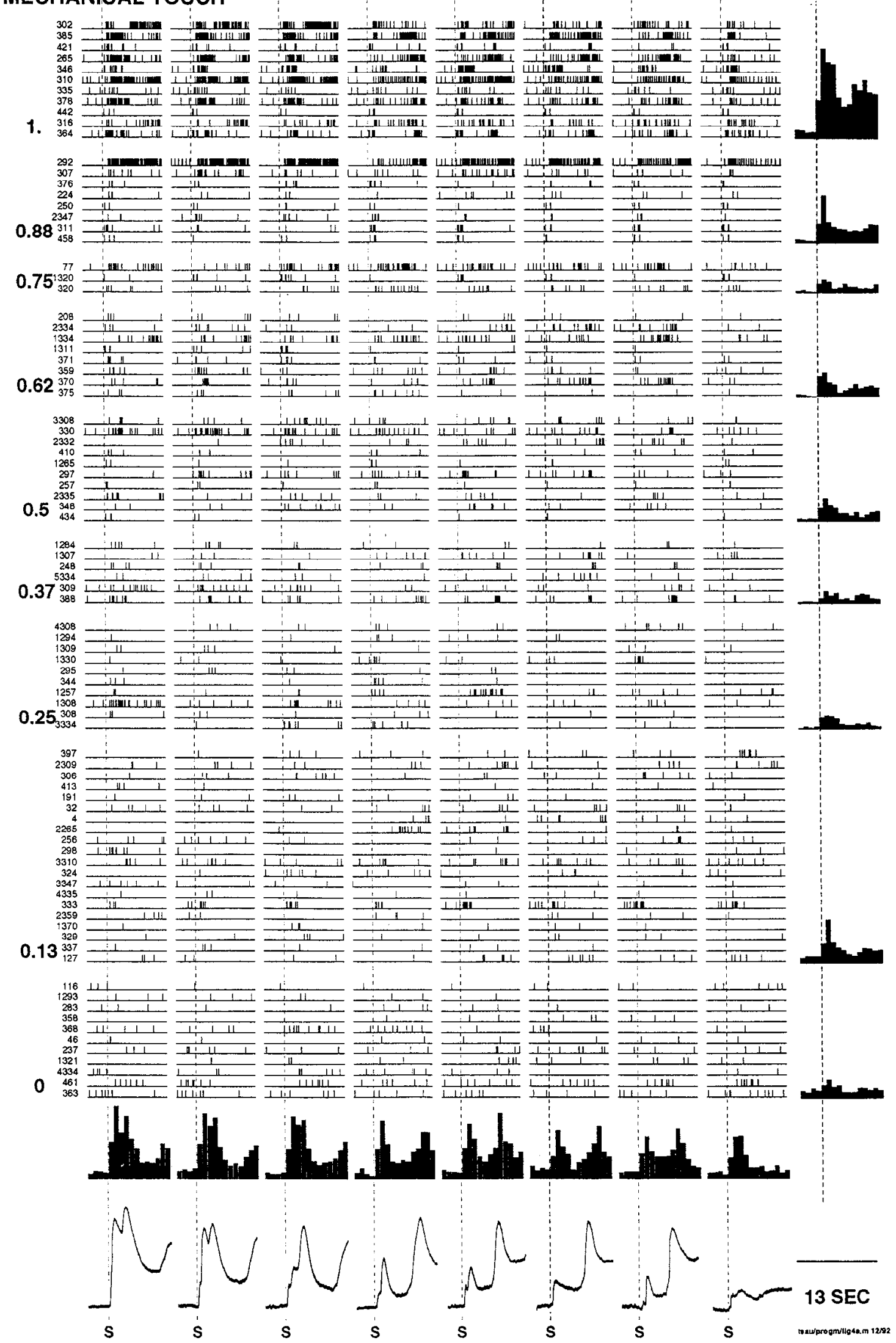

Figure 2. Raster diagrams of action potential activity detected in an Aplysia abdominal ganglion during eight presentations of a light touch separated by 10-15 min intervals. Each vertical panel shows a $13 \mathrm{sec}$ recording. Many neurons have one or more trials where the response is substantially larger (or smaller) than the mean. There are also large systematic differences between the trials. The cells are shown in nine groups according to their response probability (large numbers to the left). Histograms for each response probability group are shown on the right; these histograms include the activity of all the cells in the group. In this and similar figures histograms of spike density versus time are shown for each 
INTRACELLULAR ELECTRODE

1.

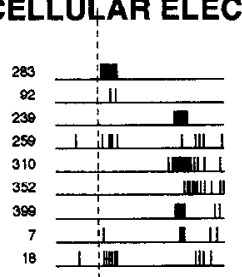

0.8

0.6

0.4

0.2

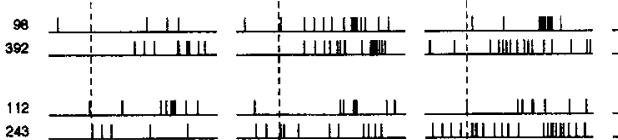

$243+11$

\begin{tabular}{l:l}
124 & \\
\hline 1346 & 1 \\
1 & 111
\end{tabular}

1346
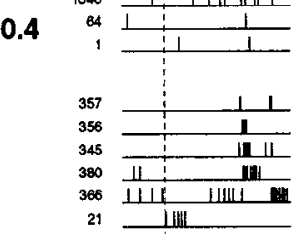

$21 \frac{1}{1}$

1002

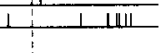

\begin{tabular}{l:l} 
& \\
260 & 1 \\
\hline 280 & 1 \\
\hline 279 & 1
\end{tabular}

it

27

115

115

613
305

305

101

34

1043
45

45
481

281

24

251

346.

321
1310
358

358

391

368

170

81

\begin{tabular}{lll}
40 \\
30 \\
\hline \\
1
\end{tabular}

24

17

1352

0
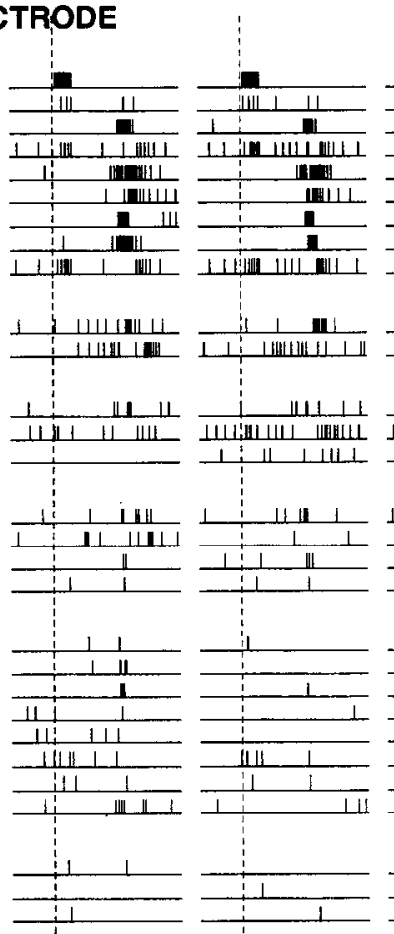

983
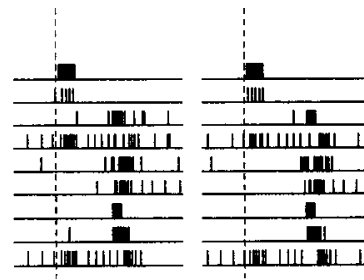

\section{(1)}
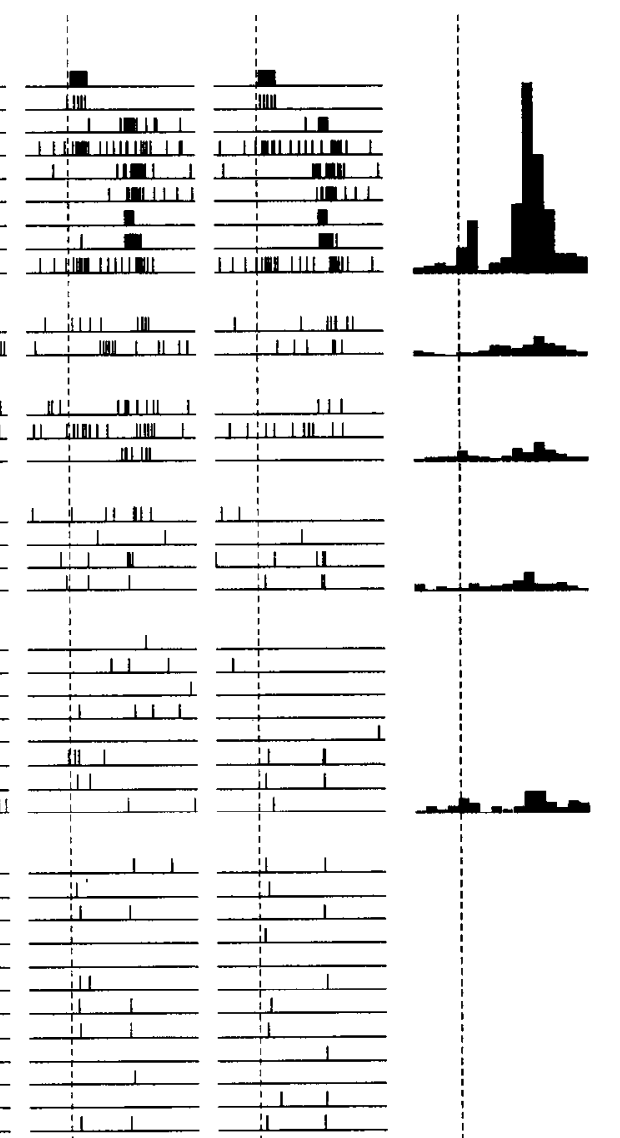

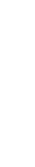
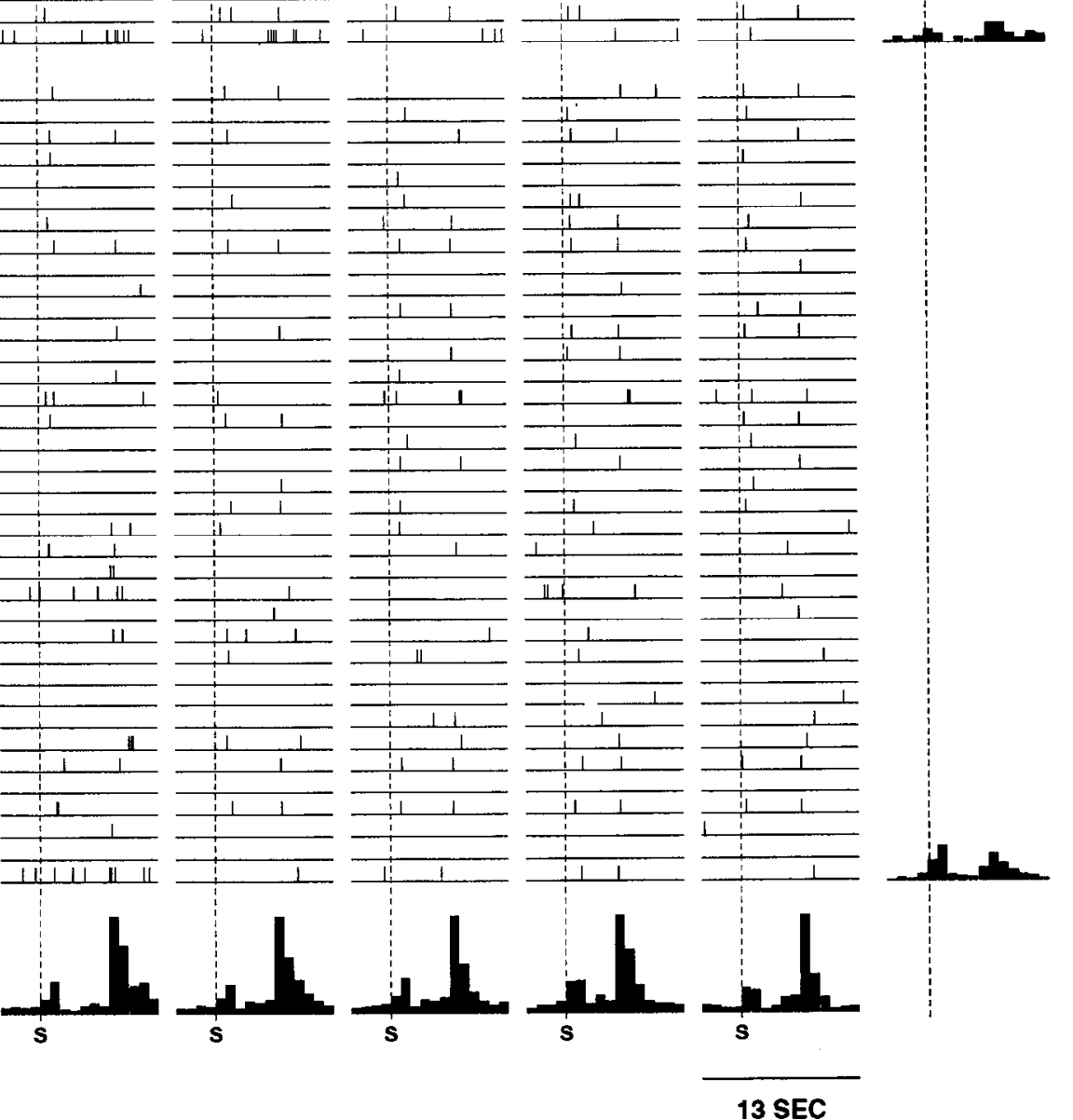

Figure 3. Raster diagrams of action potential activity in response to five microelectrode stimulations of a large LUQ neuron separated by $10-15$ min intervals. The stimulated neuron (top trace) was driven to make 19 spikes in each trial. A number of neurons have one or more trials where the response is substantially larger (or smaller) than the mean. The cells are shown in six groups according to their response probability (large numbers to the left). There were eight trials recorded in this experiment; using the paired $t$ test on spike counts across trials, three of these were significantly different and are not included in this figure. The trials in this figure were trials $1,3,5,6$, and 7 of the experiment. Irials 2,3 , and 4 of this experiment are shown in Figure 7. The microelectrode stimulation did not lead to gill contractions. This experiment was the only one of several attempted where stimulating the neuron drove activity in more than five neurons and we held the cell for more than six trials. Experiment number A217.

trial (panel) below the raster diagrams. Each bar of the histograms represents spike counts per 512 data points (0.8 sec) for all the neurons in the corresponding group of raster diagrams. The histograms at the bottom are the results from all the cells in each trial; the histograms at the right are the results from all the cells in each group for all trials. The gill withdrawals measured from the gill area on the videotape recordings are shown at the bottom. In the first trial $11 \%$ of the neurons accounted for $50 \%$ of the total number of spikes. In this and similar figures the numbers to the left of each line are identification numbers derived from their signal locations on the photodiode array; the dashed lines are placed at the beginning of the current steps that drove the mechanical toucher. For clarity of presentation 62 neurons that made less than 1 spike per trial were not included in this figure. One was from the group with a probability of $0.37,2$ were from the group with a probability of $0.25,20$ were from the group with a probability of 0.13 , and 39 were from the group with a probability of 0 . Experiment number A206; all of the trials are shown. 

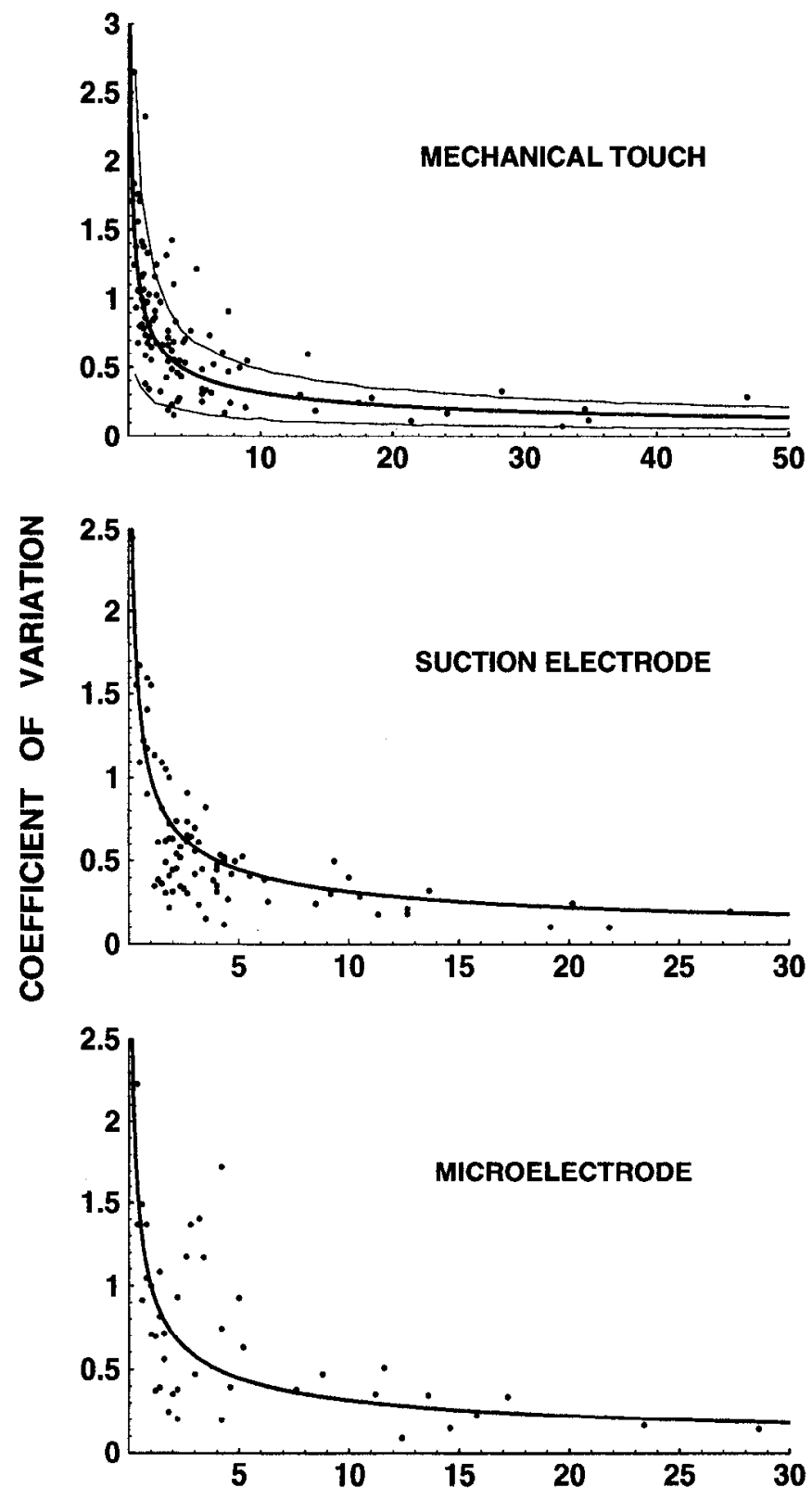

\section{MEAN SPIKE COUNT}

Figure 4. Plots of measured values of the $\mathrm{CV}$ versus the mean number of spikes for each neuron (dots) and the expected relationship for a Poisson distribution (middle solid curve in the top panel and solid curves in the bottom two panels). The top panel represents the neurons from the experiment illustrated in Figure 2 (A206), the middle panel is from an experiment using a suction electrode stimulus (A198), and the bottom panel is from the experiment illustrated in Figure 3 (A217). For all three data sets the spike counts in the total poststimulus interval were used. The $t$ test was used to remove significantly different trials in all three data sets. The top and bottom curves in the top panel represents the window where $95 \%$ of the data points would be expected to fall if the data were generated by a random selection of seven spike counts from a Poisson distribution of spike counts at each mean value. To obtain these curves we selected 1000 sets of seven spike counts from a Poisson distribution centered at each mean. We then calculated each of the CVs and determined the $\mathrm{CV}$ values at $\pm 1.96 \times \mathrm{SD}$. Thus, $2.5 \%$ of the CVs should appear above and below the window defined by the two curves if the spike counts were distributed according to a Poisson distribution and if they were chosen randomly. The $\chi^{2}$ values for the goodness-offit to the Poisson equation in the panels in this figure and in Figure 9 ranged from 6 to 17 with degrees of freedom between 57 and 148. These counts. These trials were removed from the data used for analysis in this section. Using this test the eighth trial in Figure 2 was different from the first seven. It should be pointed out that a $t$ test based on spike counts will not detect large trial-to-trial differences in the timing of activity unless the timing changes were such that some spikes were delayed past the end of the recording period. Where trials have been removed from the data set used for analysis we have indicated this fact in the figure caption. In most instances the effect of removing trials was small (but perceptible).

Effects of habituation. In many experiments, including the one illustrated in Figure 2, there was an apparent decline in the response over trials that is probably due to a slight habituation that occurs with the $15 \mathrm{~min}$ interstimulus intervals we used (Falk et al., 1993). Trial-to-trial differences that result from this habituation could confound differences that result from other sources. The following arguments suggest that the confounding effects of habituation are small. The area under the spike count histograms at the bottom of Figure 2 declines by a factor of about 2 during the eight trials. However, inspection of the responses of individual neurons suggests that they often vary by a factor much larger than 2. We determined the means and ranges of the summed spike counts (all neurons) and the spike counts of each individual neuron. Normalizing all means to 1.0 , we found that the summed activity had a range of $0.57-1.21$, a factor of 2.1. On the other hand, the mean of the ranges for the individual neurons illustrated in Figure 2 was $0.25-2.17$, a factor of 8.7. Thus, statistical measures applied to individual neurons should be dominated by the variability in the response of individual neurons rather than the confounding (but much smallcr) effect of habituation. To show that this was the case, we computed statistical measures (see below) on the original data and on data normalized for the effect of habituation; normalization had only small effects (data not shown). We also compared the statistical measures from the first seven trials of Figure 2 and from all eight trials. In terms of summed activity it is the eighth trial that has the largest decline in response. Omitting the eighth trial had only small effects (data not shown). From these results we conclude that the confounding effect of habituation was small.

Variations in response versus mean response. The mean response, the SD, and the coefficient of variation (CV) were measured for each neuron in Figure 2. These CV values are the data points in the top panel of Figure 4, where the CVs are plotted against the mean response. The CVs decrease from values of greater than 2 for small mean responses to values of about 0.3 for large means. These experimentally determined CVs can be compared with the CVs predicted from the means on the assumption that the variations follow a Poisson distribution. For a Poisson distribution the $\mathrm{SD}$ is the square root of the mean and therefore the $\mathrm{CV}$ is equal to $m^{-1 / 2}$. This relationship is plotted in Figure 4 as the middle curve in the top panel and the curves in the bottom two panels; these curves fit the data reasonably well although, as expected, there is substantial scatter in the data. The top and bottom curves in the top panel represent the window where $95 \%$ of the data points would fall if the CVs were generated from a random selection of seven spike counts from a Poisson distribution of the mean spike counts. While

values are much too low to reject the hypothesis that the data are fit by a Poisson equation. 
most of the data points fit within this window, there were 14 data points above the window and 2 below. We presume that neurons with relatively dramatic trial-to-trial differences in spike count in Figure 2 appear as the data points above the window in Figure 4. Nonetheless, it is clear from Figure 4 that the trialto-trial variations in spike count are fit reasonably well by a Poisson distribution.

The ability of neurons to discriminate between stimulus and no stimulus. Using signal detection theory (Green and Swets, 1966) and choosing the criterion response (Eq. 2) that optimizes for the maximum number of correct responses, we calculated how well each neuron could discriminate between stimulus and no stimulus. Using the result from Figure 4, that the trial-totrial response variations can be represented by a Poisson distribution, the distribution of the responses in the pre- and poststimulus periods can be calculated from the fact that the SD is the (mean $)^{1 / 2}$ of the responses during the two periods. The probability of correct discrimination is inversely proportional to the fraction of the areas that lie under both distribution curves (Eq. 3). A histogram of the mean values for the cells in all three mechanical touch experiments is shown in Figure 5 (top). A score of 0.50 represents a neuron that cannot distinguish between stimulus and no stimulus, and 1.00 represents a cell that distinguished perfectly. As can be seen, the neurons are approximately equally distributed over all values of probability of correct discrimination. The lowest scores were near 0.50 , which means that these cells could do no better than chance. The highest score was 0.9998 . For the 11 neurons shown in the top section of Figure 2 the probabilities were $0.997,0.999$, $0.967,0.996,0.980,0.938,0.851,0.958,0.931,0.945$, and 0.991 . These neurons would make a response smaller than the criterion $3,0,33,4,20,62,149,42,69,55$, and 9 times in 1000 trials.

The probability of responding in each trial. The probability of correct discrimination is based on a criterion determined a posteriori from the mean responses and therefore cannot address an issue that the animal faces, that of deciding in each trial whether a stimulus occurred. By choosing a criterion in advance (to be counted as a response the spike count had to be so large that it would occur by chance with a probability of less than 0.05 ) we could determine whether, in each trial, the neuron responded. The probabilities for different background spike counts were determined from the distribution of prestimulus counts. Each neuron received a binary score (response-no response) for each trial. The response probability of the neuron is simply the fraction of the trials that were scored as responding. The grouping of the cells in Figures 2 and 3 was done using the response probability score. The cells at the top made a response (using the criterion of 0.05 ) in every trial; those at the bottom did not make a response in any trial by that criterion. However, even in the group with 0.0 response probability, a response is detected if we sum the activity of all the cells (histograms on the left of Figs. 2, 3).

A histogram of the number of cells in each response probability category from the experiment illustrated in Figure 2 is shown in the top panel of Figure 6. As with the probability of correct discrimination, there is a wide distribution of response probabilities including the extremes of 0.0 and 1.0 . We also tested a less stringent criterion for a response $(0.10)$. The distribution of reliabilities using this less stringent criterion was similarly broad (data not shown). Neurons with perfect response probability comprise only a small fraction; the majority are less reliable.
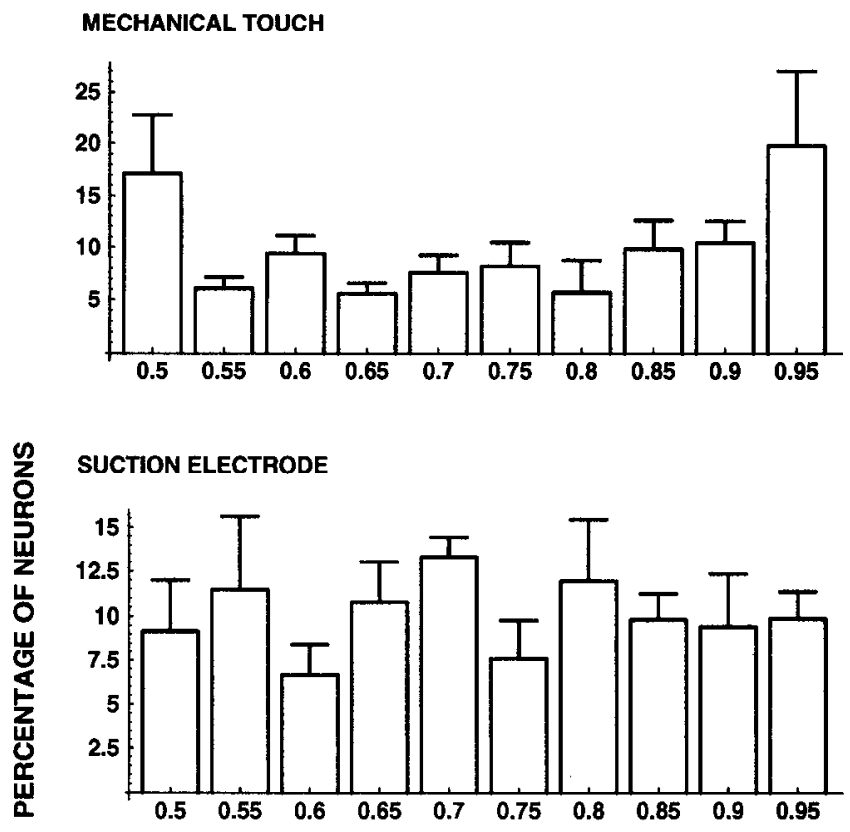

MICROELECTRODE

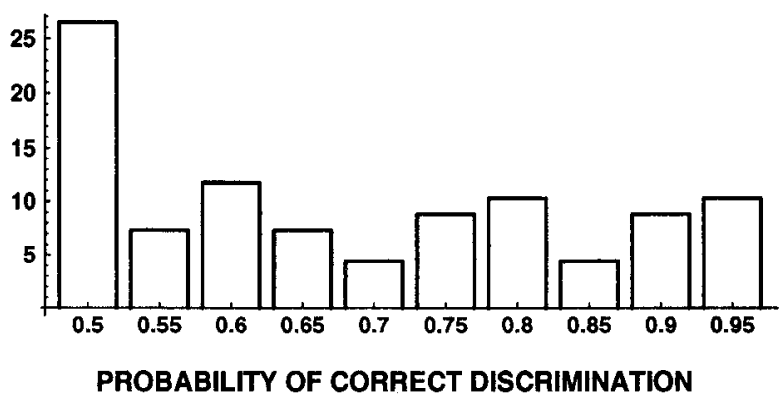

Figure 5. Histograms of percentage of neurons versus probability of correct discrimination. A value of 0.5 represents zero ability to distinguish between stimulus and no stimulus; a value of 1.0 means that the cell discriminates perfectly. For all three kinds of stimuli there is a broad distribution of probability of correct discrimination values. Each bar represents 0.05 intervals of probability; the label gives the lowest probability value for each group of neurons. Three panels are shown. The data in the top panel are the mean values from the three experiments using a mechanical touch. The data in the middle panel are the mean values from the four experiments using a suction electrode stimulus. The data in the bottom panel come from the experiment illustrated in Figure 3 (A217) using a microelectrode stimulus. Neurons with poststimulus mean responses smaller than prestimulus means were not included in the calculations.

We determined the locations on the diode array of the individual neurons in each response probability group. There was no apparent clustering of neurons from one group; all of the response probability groups had neurons in widespread locations in the ganglion.

\section{Variations that are apparently systematic}

While Figure 2 illustrates the results from all of the recorded trials, the five trials illustrated in Figure 3 were selected from an experiment where eight trials were recorded. The paired $t$ test comparing the differences indicated that the five trials in Figure 3 were not significantly different from each other. However, the additional trials not shown in Figures 3 had differences 
MECHANICAL TOUCH
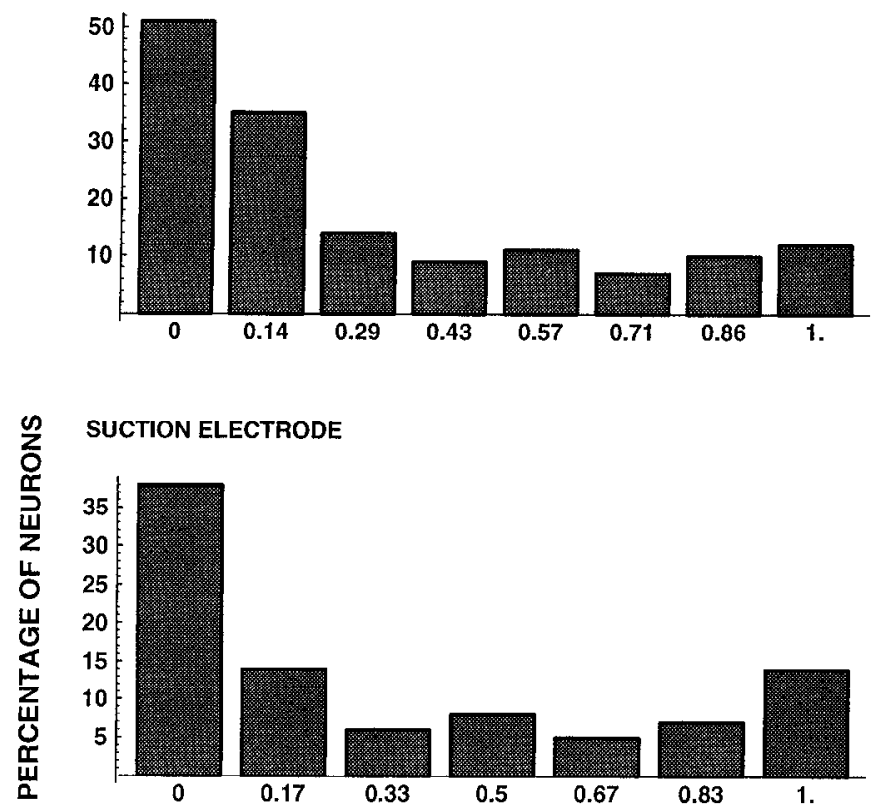

MICROELECTRODE

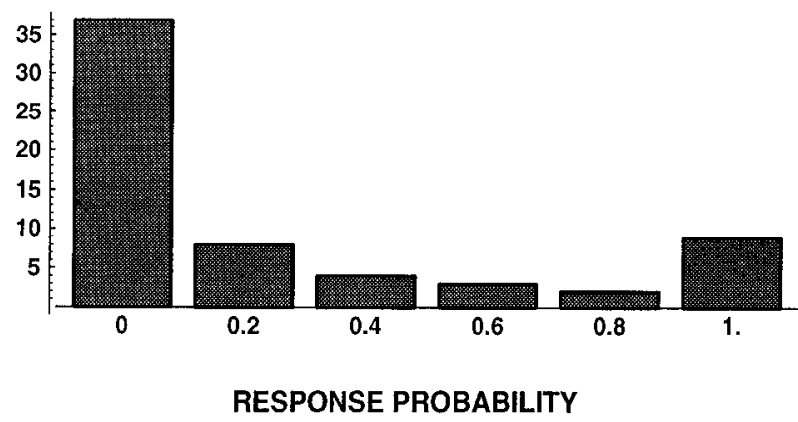

Figure 6. Histograms of number of neurons versus response probability. A value of 0.0 represents zero response probability, and a value of 1.0 is perfect response probability. The response probabilities were calculated using a criterion of 0.05 . For all three stimuli there is a broad distribution of reliabilities. Three panels are shown. The data in the top panel come from the experiment illustrated in Figure 2 (A206, mechanical stimulus); the middle panel comes from the suction electrode experiment A198; the bottom panel comes from the experiment illustrated in Figure 3 (A217, intracellular microelectrode stimulus).

in spike count that were larger, systematic, and significantly different. Figure 7 illustrates trials that are systematically different from both of the experiments. On the left are the last two trials from Figure 2 and on the right three trials from the experiment of Figure 3 . In the left section, the eighth trial lacks a late component in the response that is seen in many of the cells in the seventh trial. The recording of gill area, which continued for an additional 30 seconds (not shown), indicated that this late component is missing from the eighth trial because it was delayed past the end of the optical recording. Indeed, the timing of the delayed components, probably respiratory pumping episodes elicited by the touch (Kanz et al., 1979), becomes progressively more delayed throughout the experiment. The right section of Figure 7 contains three trials from the experiment illustrated in Figure 3. The left panel in this section (trial 3) is the second panel of Figure 3. Using the $t$ test, the response in the middle panel (trial 2) is significantly larger than the response seen in trial 3. The response in right panel (trial 4) is much smaller, with a greatly reduced second component.

In the three experiments using the mechanical toucher, six trials out of 28 were significantly different using the paired $t$ test. In the microelectrode experiment, three trials out of eight were different. In eight out of these nine instances where there were systematic differences as determined by paired $t$ test, they were associated with the presence or absence of a delayed component; only one (trial 2 in experiment A217) resulted from a simple quantitative change in spike counts.

The paired $t$ test, based on spike counts, will, however, not detect changes in the timing of activity. The histograms and gill withdrawals shown at the bottom of Figure 2 indicate that there are clear trial-to-trial differences in the timing of the delayed components of both summed spike activity and withdrawal. To look for systematic differences in time of maximum response, the time of the peak response in individual trials was compared with the mean peak time (time of peak response averaged over eight trials) for each neuron. The left panel of Figure 8 illustrates histograms of the differences between the time of peak in the indicated trial and the mean for each neuron from the experiment of Figure 2 (A206). The right panel illustrates the same measurements from another experiment (A207) with a mechanical toucher. In the results from A207, almost all of the differences from the mean fall in the two bins adjacent to the zero time. The same result was obtained even when the bin widths were reduced from $640 \mathrm{msec}$ to $80 \mathrm{msec}$. For this preparation no systematic trial-to-trial differences in the timing of peak activity were detected. Similar results were obtained for the microelectrode experiment (Fig. 3, A217). In contrast, for trials $1,2,3$, and 8 of experiment A206 (Fig. 8, left) most neurons have their time of maximum activity carlier than the mean while in trials 4-6 the majority of the neurons have their maximum later than the mean. Thus, there were substantial systematic changes in the time of maximum activity in experiment A206.

We wanted to determine whether those neurons that had the earliest response in the first trial of experiment A206 would also have the earliest response in subsequent trials. The histograms for the half of the neurons with the earliest times in trial 1 are plotted in the middle panel of Figure 8 . It is clear that the neurons selected for having the earliest response in trial 1 do not continue to be the earliest responders in trials $2-8$. In trials 2-8 they have differences that are indistinguishable from the complete set of neurons. Thus, having an early response in the first trial did not mean that the neurons would also have an early response in subsequent trials. Even though there is a systematic shift in time of maximum activity, an individual neuron's position in the shifting response was not fixed.

If we use restricted time intervals for determining spike counts, the systematic differences in the timing of activity can also be detected in increased CVs of the spike counts. Figure 9 shows plots of CVs versus mean spike count for two intervals, 3.25$6.5 \mathrm{sec}$ (just following the stimulus) and 9.75-13 sec (at the end of the recording), in the experiment of Figure 2. In contrast to the result from the entire poststimulus interval from this experiment (Fig. 4, top) and for the immediately poststimulus interval (Fig. 9, bottom), most of the data points for the late interval fall above the curve predicted from a Poisson distribution (Fig. 9, top). Thus, the systematic effects have increased the CV of spike counts over that predicted by a Poisson distribution. 


\section{A206}

TRIAL 7

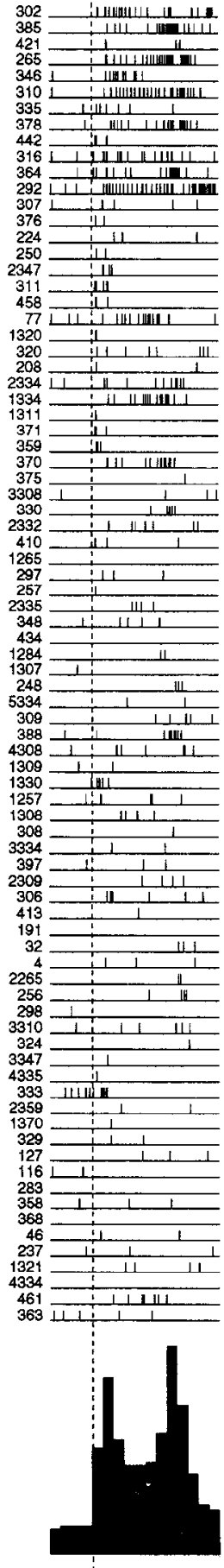

s
TRIAL 8

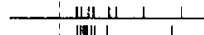

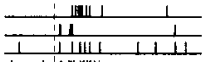
1.

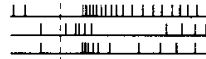

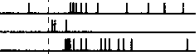

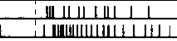
$\frac{I^{\frac{11}{11}}}{\frac{111}{11}}$

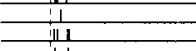
$\frac{11}{111111}$ \begin{tabular}{l:l}
\hline 1111 \\
\hdashline-111 \\
\hline
\end{tabular}

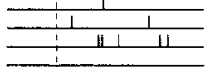
$\frac{\frac{1}{1}}{\frac{111}{1 ! ! 11}}$

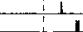
$\frac{111}{111}$ ב $\frac{1}{1-1}$ $\rightarrow+1$ $\frac{1}{111}$ \begin{tabular}{l|l}
\hline & 1 \\
\hline 1 & 1 \\
\hline & 11 \\
\hline & 1
\end{tabular} \begin{tabular}{l:l}
\hline & 1 \\
\hline 1 & 1 \\
\hline & 1
\end{tabular} $\begin{array}{r}1 \quad 11 \\ \hline 1 \quad 1 \quad 1 \\ \hline 1 \\ \hline 1\end{array}$ IIس1

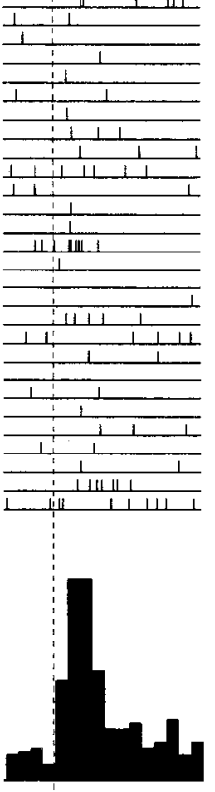

s
TRIAL 3

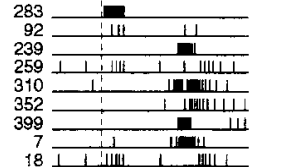

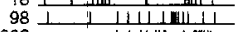

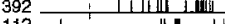

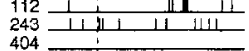

$124 \frac{1 ! 1}{1346}$

\begin{tabular}{rl|l}
64 \\
1 \\
\hline & 1 \\
\hline
\end{tabular}

${ }_{345}^{357} \frac{11}{11}$

${ }_{366}^{380}{ }^{11} 1 t^{4} 111-$

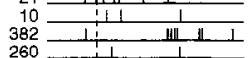

${ }_{279}^{260}=\frac{1}{1}$

325
104
115

${ }^{60}=\frac{1}{305}$

${ }_{84}^{101}=\frac{1}{1}$

34
1043
45

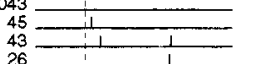

$2 6 \longdiv { 1 } \frac { 1 } { 1 . 1 }$

346

$358=$

369
391
368
179
81

179
81
40
30

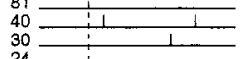

\begin{tabular}{l}
24 \\
\hline 1 \\
\hline
\end{tabular}

$1 3 9 2 \longdiv { \square }$

${ }_{393}^{403}$

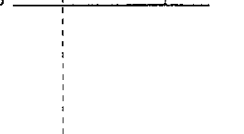

TRIAL 2
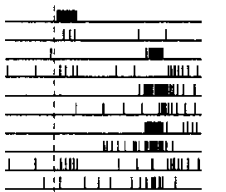

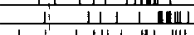
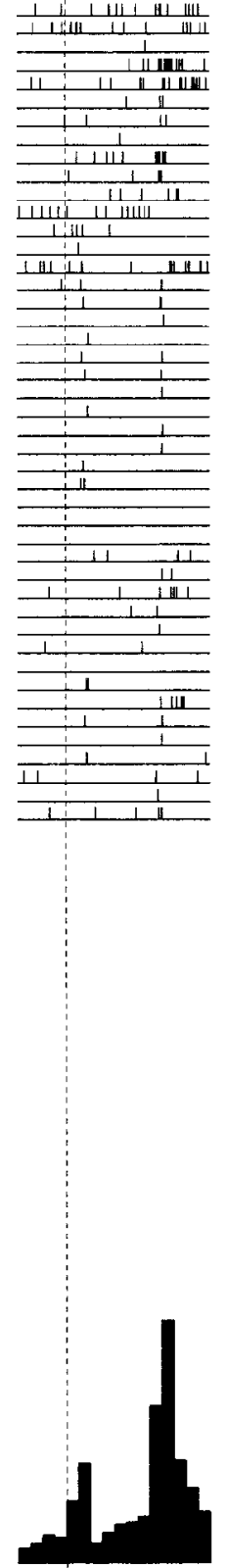

s
TRIAL 4
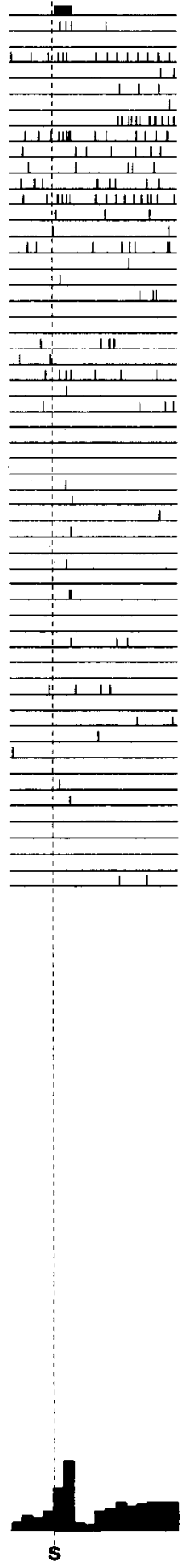

Figure 7. Raster diagrams of action potential activity from two preparations illustrating large systematic trialto-trial differences. On the left are two trials from Figure 2(A206); on the right are three trials from the experiment also illustrated in Figure 3 (A217). In these sets of trials, many neurons show large and similar changes from trial to trial. The first panel in the right section is the third trial in the experiment (and the second panel of Fig. 3). The middle panel is the second trial and the right panel is the fourth trial in the experiment.

\section{Comparing the three kinds of stimulation}

While we think that the mechanical stimulus was reproducibly applied from trial to trial, as a control for possible effects of stimulus variation two additional kinds of stimulation were used: electric shock to the siphon skin via a suction electrode, and intracellular electrode stimulation of an individual neuron in the abdominal ganglion. Since the position of the suction electrode is held fixed throughout the course of the experiment, this kind of stimulation may be more consistent in terms of location on the siphon skin. With the microelectrode stimulation of an individual neuron in the abdominal ganglion, exactly 19 action potentials were elicited in each trial, eliminating the possibility that the trial-to-trial variability from touch or suction 
Figure 8. Histograms of the differences in the time of peak response in each trial and the mean time for all trials for each neuron in two experiments. These differences are relatively large and they change systematically from trial to trial in experiment A206 (left and middle panels). The differences are small and there is no apparent systematic change in experiment A207 (right pan$e l)$. For A207 almost all of the differences from the mean time of the peak fall in the two bins adjacent to the mean time (shown as zero). In the middle panel, the histograms for the half of the cells with the earliest response in trial 1 of A206 (left panel) are shown. Although these neurons are the earliest responders in trial 1 , in all subsequent trials their time differences are similar to the differences seen for all cells (left panel). Thus, they are not consistently earliest. When a similar set of histograms using only the earliest neurons in the first trial was made for the data in the right panel (A207), they also did not remain consistently early in trials $2-8$. Raster diagrams of the data from experiment A207 appear in Figure 4 of Tsau et al. (1994). Eight trials are shown in each panel; the trial numbers are given on the left.

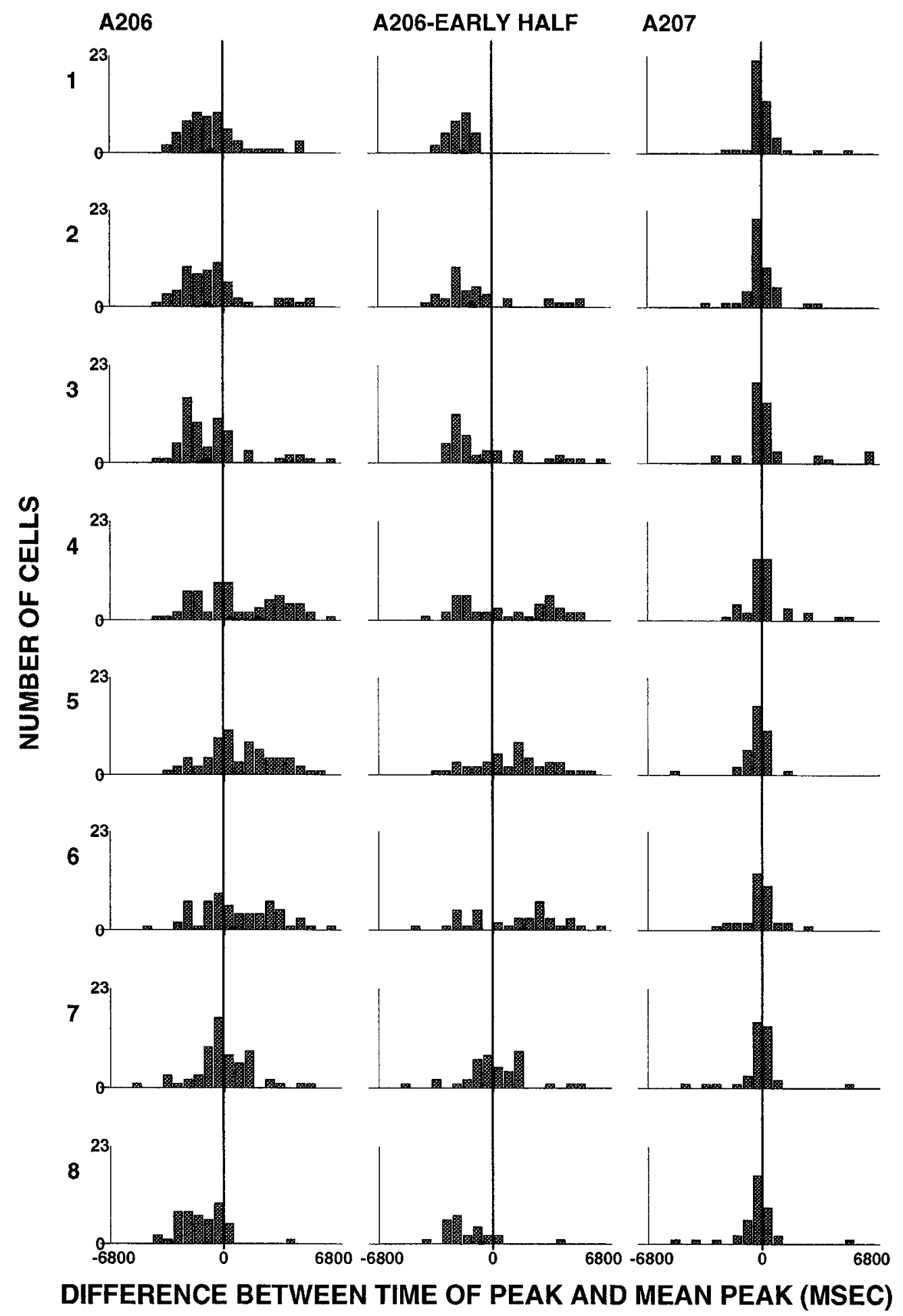

Plots of CV versus the mean response from experiments where suction electrode and microelectrode stimuli were used are shown in the middle and bottom panels of Figure 4 . The measured $\mathrm{CVs}$ are again reasonably well fit by the curve calculated on the assumption that the trial-to-trial response variation follows a Poisson distribution. The probability of correct discrimination was also calculated for each neuron in the four suction electrode experiments and the histogram of the mean values is shown in the middle panel of Figure 5. The histogram for the microelectrode experiment is shown in the bottom panel. The histograms electrode stimuli resulted from variability in the stimulus or in the process of sensory transduction. The raster diagram for five trials using the intracellular electrode stimulus is shown in Figure 3 . In each of the trials the driven cell (top trace) made 19 action potentials during a $1.3 \mathrm{sec}$ train. Evidence suggesting that the stimulated neuron may be L10 is given in Materials and Methods. The histograms of the activity of responding neurons are shown at the bottom of Figure 3 . While some neurons respond to the driven neuron during the stimulus train, the largest response occurs about $5 \mathrm{sec}$ after the stimulus. 
of response probability scores from one suction electrode experiment and the microelectrode experiment are shown in the middle and bottom panels of Figure 6 .

Comparison of the three panels in Figures 4-6 shows that the three kinds of stimuli result in CVs, probabilities of correct discrimination, and response probabilities that are similar. All three kinds of stimulation resulted in similar response variabilities.

\section{Is the behavioral output matched by the activity of individual neurons?}

The bottom two panels of Figure 2 show that the behavioral output (gill movement) and the summed activity of all the neurons (histograms) are highly correlated. Would the activity of individual neurons also be highly correlated with the behavior? Histograms of the activity of the top 30 individual neurons from Figure 2 are shown in Figure 10. In this figure the histograms are normalized to the same peak height in all trials to emphasize the timing of spike activity. The bottom histogram of Figure 10 again shows the summed activity of all of the neurons from Figure 2. Few, if any, of the individual neurons have activity patterns that match the summed activity at the bottom. The best match is neuron 378 (eighth from the top), which, compared to the summed activity, has relatively little late activity in trials $1-3$ and relatively little early activity in trials 6 and 7 . Furthermore, none of the additional 119 neurons from the experiment of Figure 2 that are not illustrated in Figure 10 had activity patterns similar to the summed activity.

However, the summed activity of several of the response probability groups was similar to summed activity of all neurons. These were response probability groups $1.0,0.62,0.5$, and 0.13 . The summed activity of the group with lowest probability, 0.13 , is shown in Figure 10 just above the summed activity of all neurons. The histograms of the summed activity from the response probability group 0.13 and the summed activity from all neurons are quite similar. Thus, the activities of groups of neurons, but not individual neurons, are highly correlated with the behavioral output.

The results from other preparations would be different. In preparation A207 (Fig. 8, right) all of the neurons are active nearly simultaneously, and thus, for this animal, there was a good correlation between the activity of many individual neurons and the behavior.

\section{Animal-to-animal differences}

Animal-to-animal differences in the response to siphon stimulation appear to be substantially larger than the differences between trials in one preparation. The three panels in Figure 11 illustrate the responses of three different preparations to a 2.0 gm touch to the siphon skin. Raster diagrams are shown at the top, and histograms of total spike count in all cells are shown at the bottom. For animal 1 most of the cells make a very brief response immediately following the stimulus, while for animal 3 many of the cells remain active for the remainder of the recording period. The response of the neurons of animal 2 is intermediate. These differences are reflected in the spike count histograms at the bottom.

The number of neurons detected in the three recordings of Figure 11 also differ. However, these differences might be explaincd by animal-to-animal differences in the effectiveness of the dye staining. Thus, we do not know if there actually was a
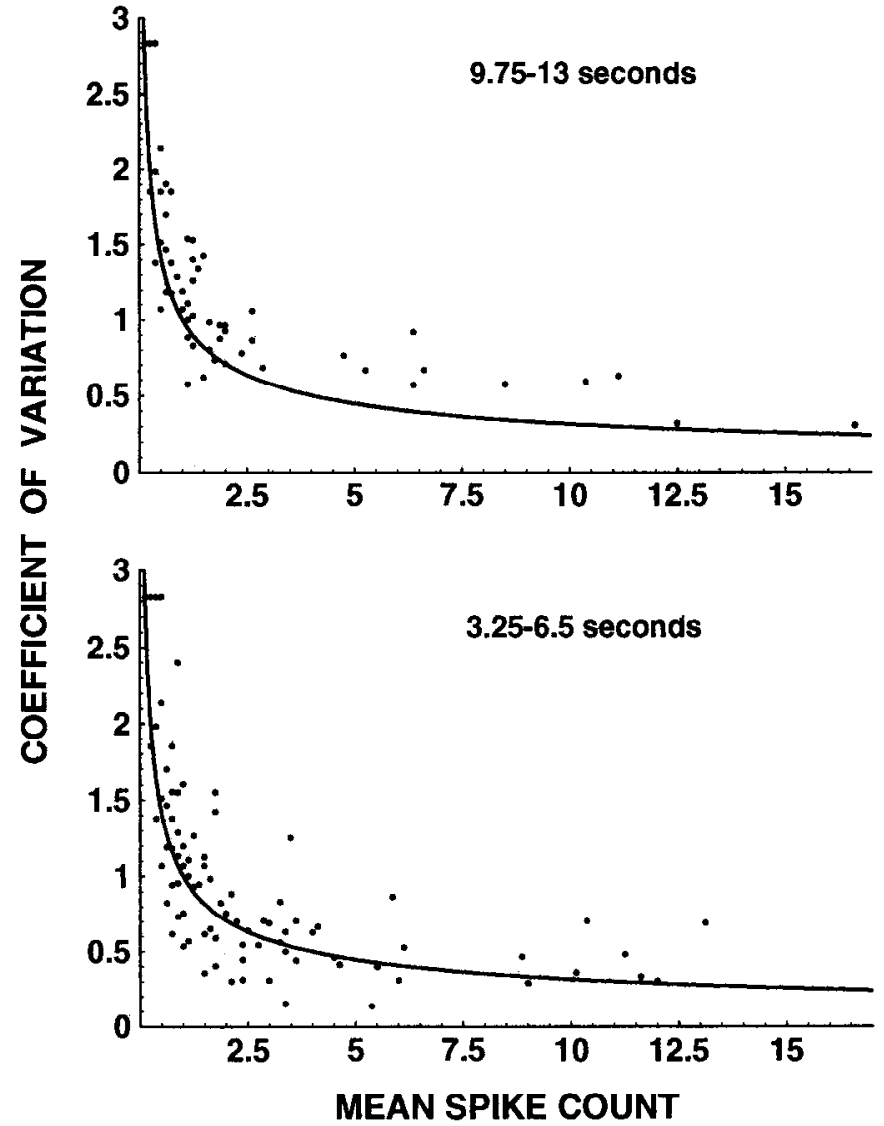

Figure 9. Plots of measured values of the $\mathrm{CV}$ versus the mean number of spikes for each neuron (dots) and the theoretical relationship for a Poisson distribution (solid curves). The top panel represents the spike counts for the interval between 9.75 and $13 \mathrm{sec}$ and the bottom panel represents the counts for the $3.25 \mathrm{sec}$ interval immediately after the stimulus. For the later interval many of the data points fall above the predicted curve, indicating that the systematic effects that led to differences in spike timing also led to increased CVs of spike counts. Experiment A206.

different number of neurons responding in the three different preparations.

To illustrate the differences in time course of the response in different animals we plotted the time integral of the response (the integral of the summed spike count from all the neurons). The bottom panel of Figure 12 shows such a plot for 24 preparations; the top panel illustrates the same plot for six trials from one animal. The results from one animal are more tightly grouped than the results from different animals. We measured the number of seconds it took for the time integral to go from $0 \%$ to $80 \%$ of its maximum value for 30 preparations. A histogram of these times is shown as the solid line in Figure 13. There is a range of a factor of six between the fastest and slowest responding animals. The $80 \%$ times for the six trials from the animal shown in the top panel of Figure 12 are shown superimposed as the hatched area in Figure 13. Again, these times have a narrower distribution than the times from different animals.

The recordings from different animals also differ in the distribution of spikes among neurons. In some preparations a few neurons contribute a large fraction of the spikes while in other preparations the number of spikes is more cvenly distributcd among neurons. The top section of Figure 14 illustrates the 
Figure 10. Histograms of spike times for the 30 neurons from the top of Figure 2 (top section), the summed spike time of the neurons from the response probability group 0.13 of Figure 2 (next to bottom row), and the summed spike times of all neurons from Figure 2 (bottom row). While few, if any, of the individual neurons have activity patterns that are well correlated with the behavioral output, the summed activity of several groups, including that with a responsc probability of 0.13 , is well correlated with the behavior. Experiment A206.

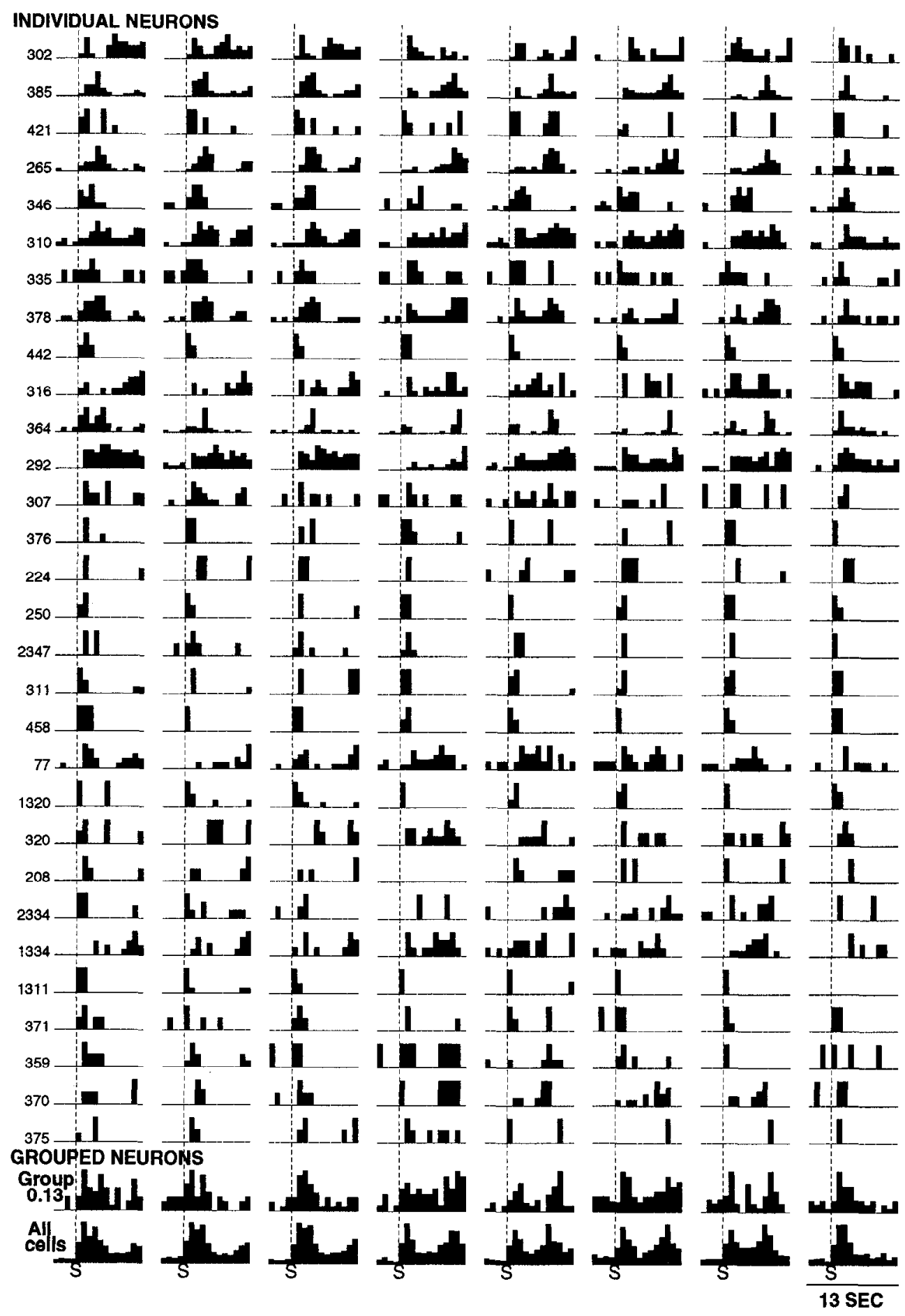

distribution of spikes among neurons in 12 animals. In the top row are three preparations where a few neurons had a large number of spikes and thus made large contribution; each of these preparations had more than seven neurons with more than 11 spikes. In the bottom row of this section are three preparations where no neurons made more than 11 spikes; the distribution of spikes among cells was much more even. The bottom section of Figure 14 shows the distribution of spikes in three trials from a single animal. While the distribution differs from trial to trial, this difference is smaller than the range of differences seen from animal to animal. Thus, the trial-to-trial differences are smaller that the animal-to-animal differences. We determined the percentage of neurons that accounted for $50 \%$ of the spikes in each trial of Figure 14. This number is shown in the figure. In the three preparations shown in the top row, $10 \%$ of the neurons account for $50 \%$ of the spikes while in the preparations shown in the bottom row of the top section, $25 \%$ of the neurons account for $50 \%$ of the spikes.

It is clear from comparison of the raster diagrams in Figures $2,3,7$, and 11 that there are additional kinds of differences in response from animal to animal. First, the consistency of the time of maximum activity can differ dramatically from animal 
ANIMAL 1

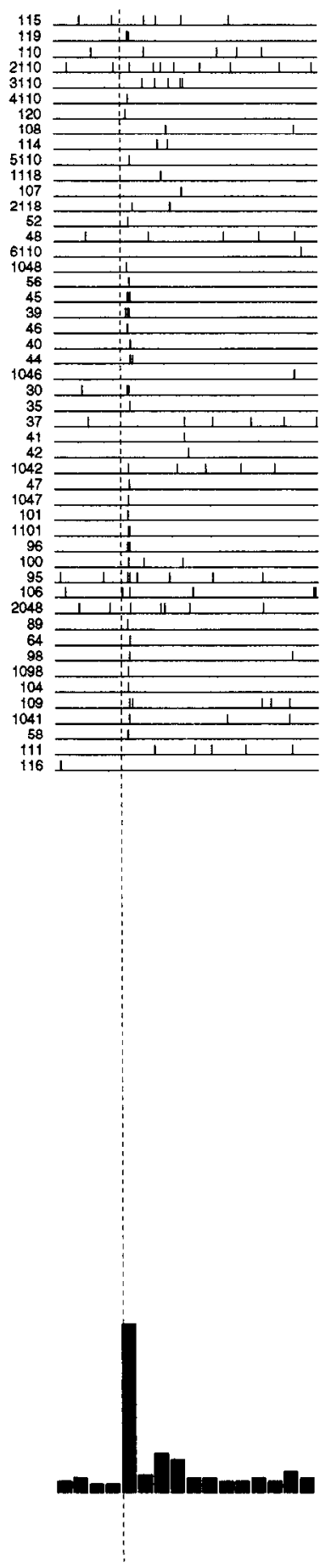

ANIMAL 2

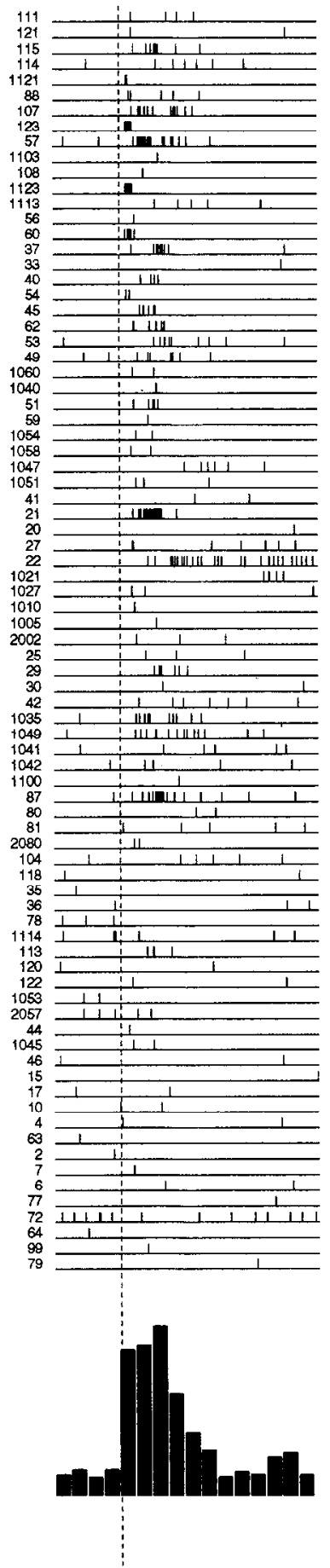

ANIMAL 3

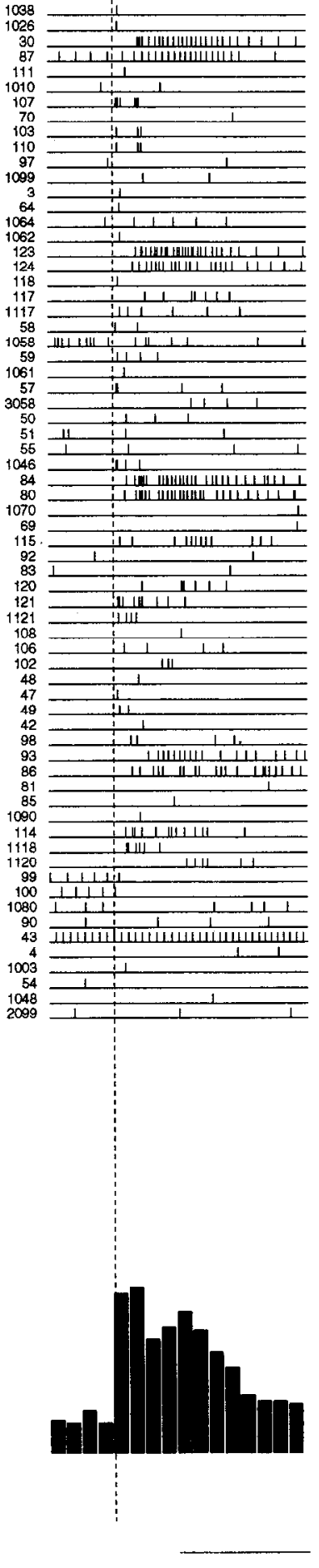

4 SEC
Figure 11. Large animal-to-animal differences in the response to a mechanical touch. The three panels come from three different preparations. The response of animal 1 is brief, the response of animal 3 is relatively prolonged, and the response of animal 2 is intermediate. The percentage of neurons that accounted for $50 \%$ of the total number of spikes was $20 \%$ for animal $1,17 \%$ for animal 2 , and $15 \%$ for animal 3. Each vertical panel is an $8 \mathrm{sec}$ recording. The brief response of most neurons from animal 1 suggests that many of these neurons may belong in Group I of the categorization of Falk et al. (1993). to animal (Fig. 8). In some animals the peak of activity is rigidly locked to the stimulus; in others there is a large trial-to-trial variability. Second, the response sometimes has one and sometimes two or more components; the two components of the response are relatively well separated in some trials for the animal illustrated in Figure 2 but not so well separated in Animal 2 of Figure 11. Thus, individual Aplysia differ in their response to the touch in many ways.

\section{Correlation between response measures and mean spike count}

Evidence presented earlier suggests that neurons with larger spike counts have more consistent responses. Figures 4 and 9 show that the CVs of spike counts were, on average, smallest for cells with a larger mean number of spikes. A result in the same direction was also found for the CVs of the time of maximum 


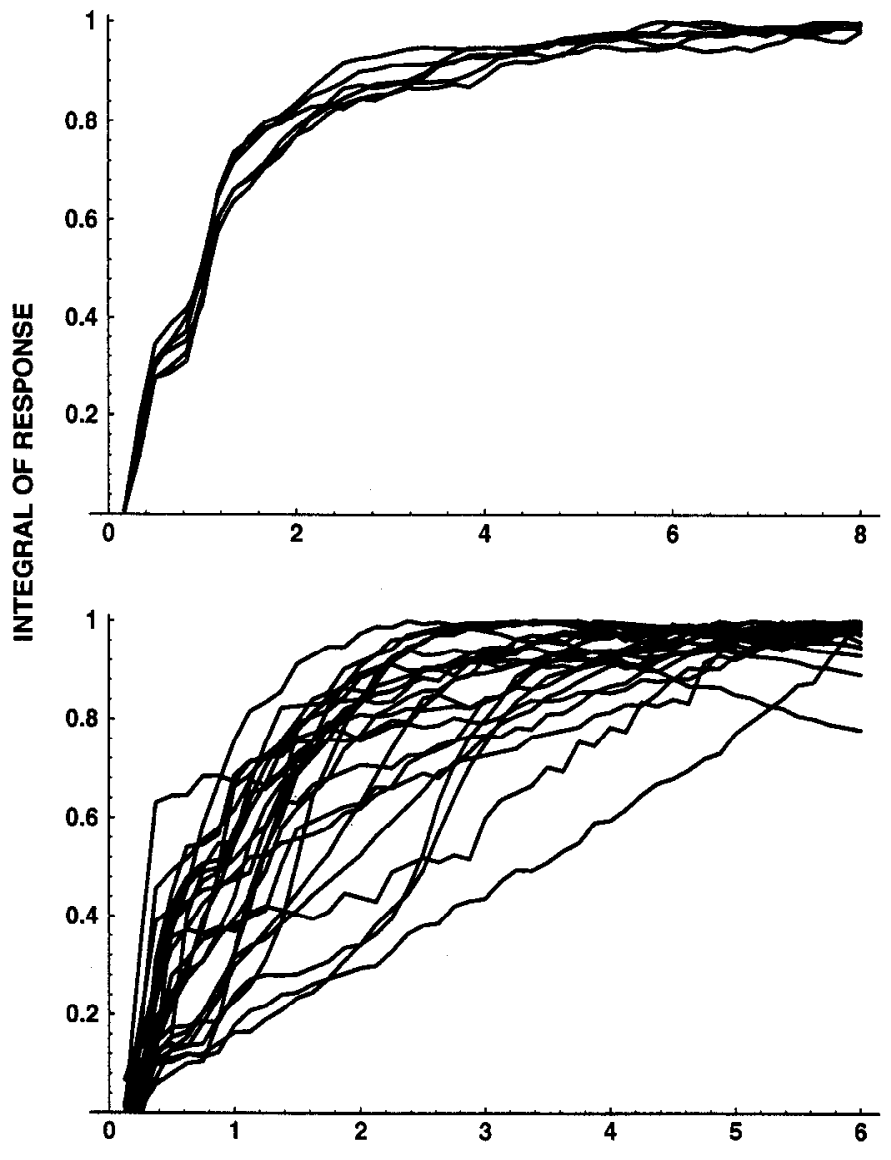

TIME AFTER STIMULUS (SEC)

Figure 12. The time integral of the response to a mechanical touchthe integral of the difference in spike counts in the poststimulus interval and the mean activity summed over all neurons in the prestimulus interval. The results for six trials from one animal, A207, are shown in the top panel. These six trials were not systematically different using the paired $t$ test. Results from 24 different animals are shown in the bottom panel. All 24 curves were from $8 \mathrm{sec}$ recordings using the 124 element photodiode array.

response (data not shown). Furthermore, inspection of Figures 2 and 3 show that there was a positive correlation between response probability score and the mean spike count. In addition to the positive correlation for individual neurons, there was also a positive correlation ( $r=0.42, p=0.05, n=8$ ) between the percentage of cells in each animal with a high probability of correct discrimination and the average spike count per neuron. Animals with a larger average response to the stimulus also have a larger percentage of cells with a high probability of correct discrimination.

\section{Discussion}

There are serious difficulties in correlating optically detected neurons with previously identified neurons. First, the number of identified neurons, while large, is only a fraction of the total number of neurons. Approximately 230 neurons in the ganglion have been identified (J. Koester, personal communication) out of a total of about 900 neurons. Thus, under the simplest assumptions, only $25 \%$ of the neurons in the optical recordings would belong to the class that have been identified. Most $(75 \%)$ have not yet been identified. Furthermore, the vast majority of

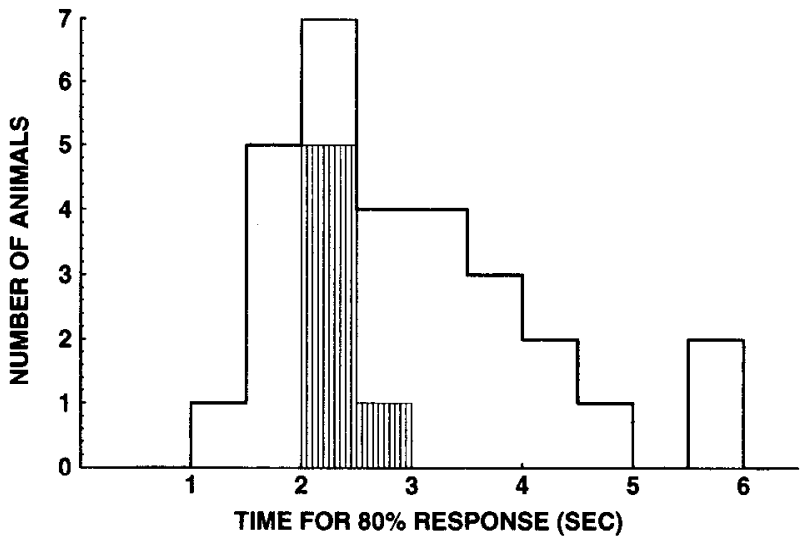

Figure 13. The distribution of rise times (0-80\%) for 30 animals (solid line) and for six trials in one preparation (hatched inset, A207). The rise times for different animals are much more widely distributed than those from the same animal. The 30 animals include the 24 illustrated in Figure 12 plus an additional six preparations whose activity was measured using the 464 pixel array.

the identified neurons can be identificd only after microclcctrode impalement. Only six neurons can be unambiguously identified by visual inspection alone (J. Koester, personal communication). In addition, many identified neurons are not in the outer layer of cell bodies and other cell bodies have to be pushed aside in order to reach these neurons. Finally, many identified neurons are not accessible to microelectrode impalement without turning the ganglion over. Thus, it will be difficult to obtain a comprehensive map of identified neurons while keeping an intact ganglion in one position.

On the other hand, except for a small number of very large neurons, it is not easy to attribute neurons detected in optical recordings to neurons observed visually (London et al., 1987). With the numerical apertures that we used, the optical recordings detect neurons throughout the thickness of the ganglion (Salzberg et al., 1977). While individual optical recordings provide the information about neuron location in the $x-y$ plane (object plane of the microscope), they give no information about the position in the $\mathrm{z}$-axis (dorsal-ventral). Although this information might be obtained from repeated optical recordings at several focal positions, trial-to-trial variability (this article) would complicate the analysis. Thus, both the difficulty in obtaining a map of identified neurons and the difficulty of determining the three-dimensional location of optically detected neurons means that an extensive comparison of previously identified neurons and those recorded optically will not be easily achieved.

\section{Trial-to-trial differences}

We have measured the consistency of the response of neurons in the Aplysia abdominal ganglion. Since the best recordings monitored the activity of $50 \%$ of the neurons in the ganglion, it seems likely that we have a reasonably complete view of the variability, the probability of correct discrimination, and response probability of Aplysia neurons to this kind of stimulus; it is not likely that additional neurons will be found with a much higher consistency.

Three kinds of stimulation were used. For the mechanical touch and the suction electrode stimulation it was possible that differences in stimulus presentation from trial to trial or differences in the response of the sensory neurons might account for the response variability. However, for the microelectrode stim- 
12 ANIMALS
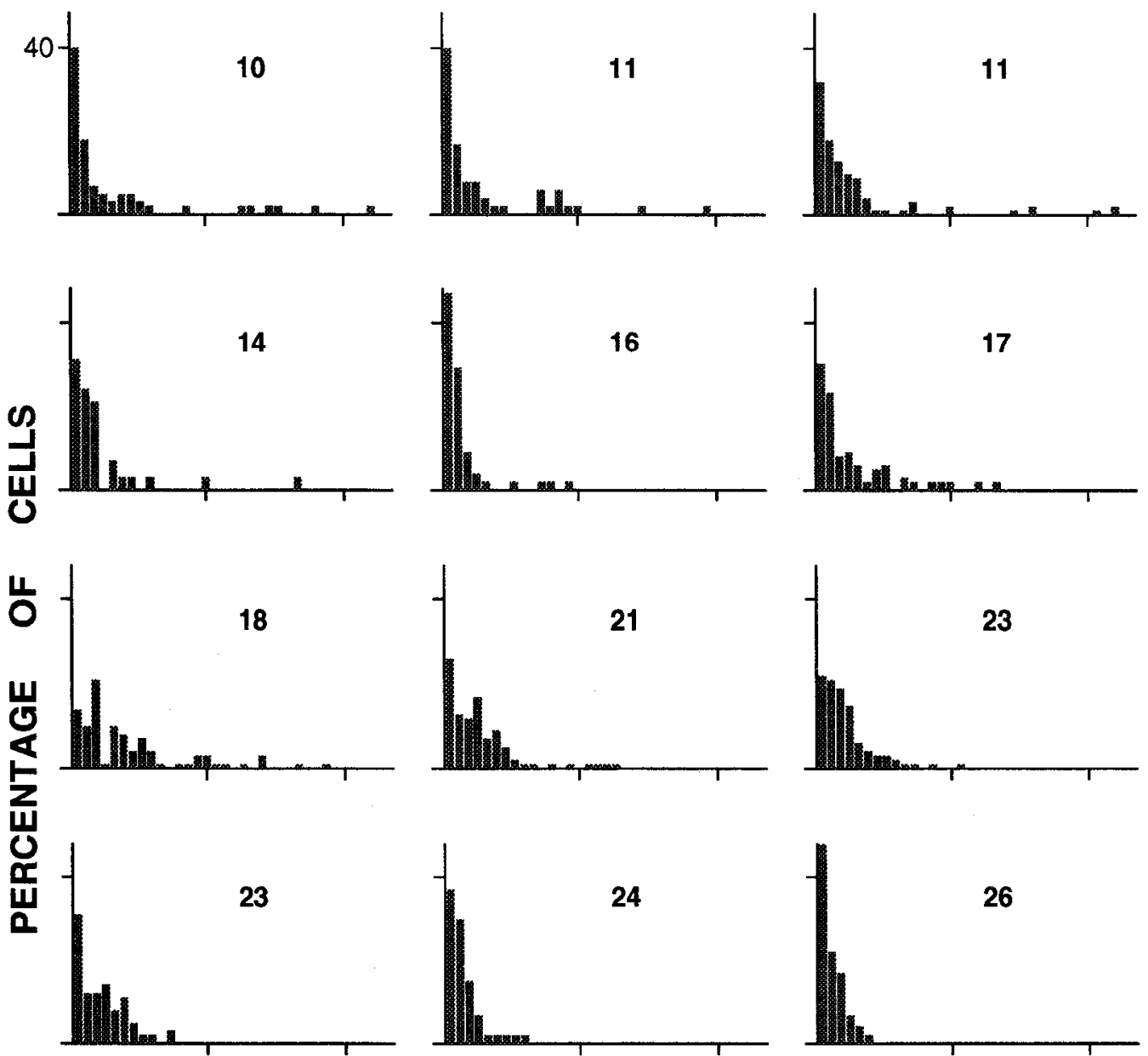

\section{TRIALS, ONE ANIMAL}
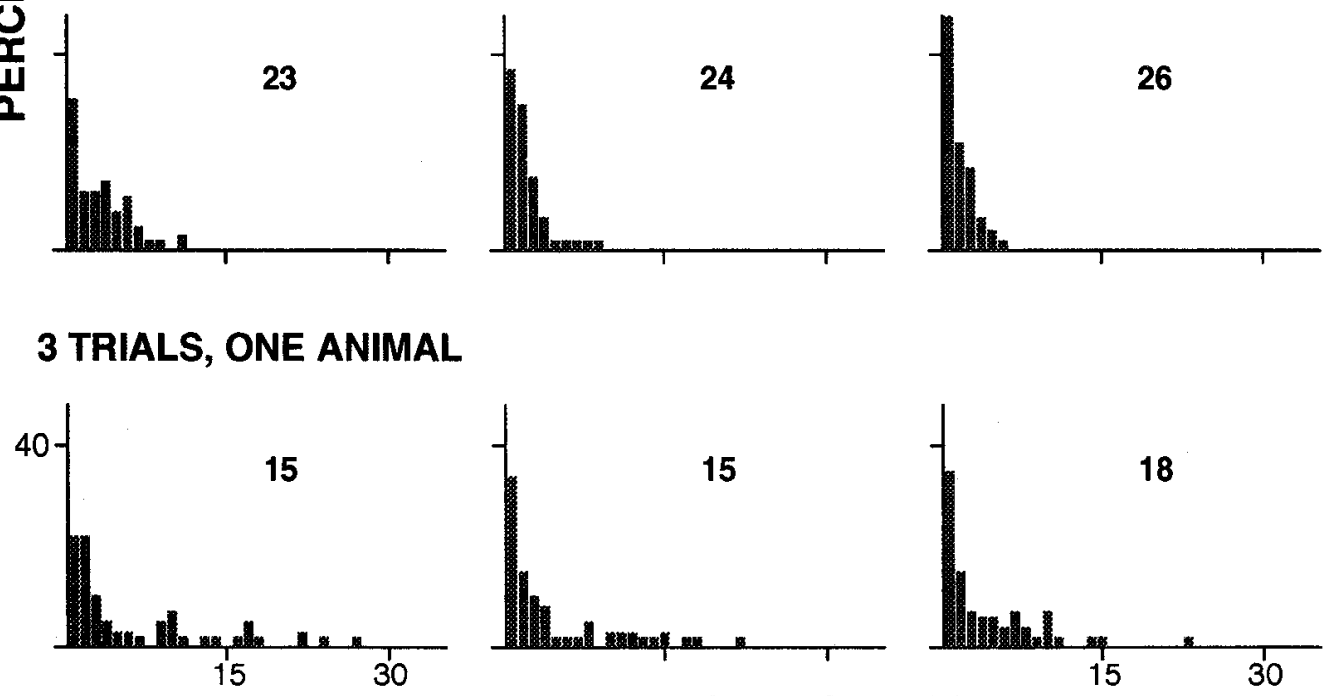

\section{NUMBER OF SPIKES}

Figure 14. Top, Histograms of the distributions of spike counts among neurons in 12 different animals. Some animals (top row) have a few neurons with a large number of spikes while in other preparations (e.g., bottom row) the distribution of spikes among neurons is more even. The result from the first trial from Figure 2 (A206) is shown in the upper right panel. This was a preparation where a few neurons accounted for a relatively large proportion of the activity. Bottom, Histograms from three trials (trials 1, 4, and 7) from one animal (A207). The differences in these three histograms are smaller than the differences that can be seen from animal to animal. Raster diagrams of the data from experiment A207 appear in Figure 4 of Tsau et al. (1994). The percentage of the neurons that account for $50 \%$ of the total spike count is indicated in each pancl. The number of cells was normalized to 100 for each panel; the number of spikes is the spike count in the 3072 data points following the stimulus.

ulus the input to the CNS was exactly the same from trial to trial in terms of number of spikes in the train. Since the trialto-trial variability of the responses to all three kinds of stimulation was similar, we conclude that the variations do not result from trial-to-trial differences in stimulus presentation. These variations must have an origin within the CNS.

There are well-understood sources of random variability. First, the probability of release of transmitter quanta is not constant but follows a Poisson distribution (del Castillo and Katz, 1954). Second, there is encoding noise that results from the threshold properties of neurons. When the membrane potential is near the spike threshold, the response is all-or-none; in this potential range the response does not accurately reflect the input and is subject to variability due to membrane potential noise. Because of these kinds of noise, it is clear that the response of a nervous system to a constant stimulus cannot be perfectly consistent. 


\begin{tabular}{lllll}
\hline $\begin{array}{l}\text { Table 1. } \\
\text { ganglia }\end{array}$ & Means, SDs, and CV of neuron number in invertebrate \\
Species & Ganglion & $\begin{array}{l}\text { Mean } \\
\text { number }\end{array}$ & SD & CV \\
\hline C. elegans & (Whole animal) & 302 & $(0)$ & $(0)$ \\
H. medicinalis & Segmental & 395 & 4.0 & 0.01 \\
H. ghilianii & Segmental & 375 & 2.3 & 0.006 \\
N. inermis & Buccal & 200 & 40 & 0.20 \\
A. californica & Abdominal & 640 & 177 & 0.28 \\
\hline
\end{tabular}

However, we do not know if these sources of noise are large enough to account for the trial-to-trial differences we measured. This question might be explored by examining response variability in realistic models of small groups of interacting neurons. For example, one might begin by determining the response fluctuations expected in the simplest two neuron model for a monosynaptic reflex. The model could then be made more realistic by assuming that many sensory neurons would have to be active to generate spikes in the motor neuron. In addition, one could add interactions with various kinds of excitatory or inhibitory interneurons and examine the effects of each of these additions on response variability.

The random differences often result in striking trial-to-trial differences in spike count for individual neurons. Nonetheless, these striking differences are only slightly larger than those predicted from the hypothesis that the responses have a Poisson distribution (Fig. 4). In apparent contrast, the probability of correct discrimination for individual Aplysia ncurons was as high as 0.9998 . However, this measure only indicates how often the neuron would be scored as giving the correct response in a binary choice situation where "maybe" is not a possible answer. It seems possible, or even likely, that the behavior of the animal will depend, in a graded way, on the number of action potentials in the response. Furthermore, the neuron with a probability of 0.9998 was unusual. On average, the 11 neurons in the top section of Figure 2 would not reach the criterion in 40 out of 1000 trials. One method for obtaining a more reliable behavioral output in the face of random noise in the spike counts of individual neurons is to make the output depend on the summed activity of several neurons.

A second source for trial-to-trial variability is intrinsic rhythmic activity in the preparation. A well-known intrinsic rhythm generated by the abdominal ganglion is the respiratory pumping rhythm (Byrne and Koester, 1978; Kanz et al., 1979), which had a period of between 3 and $60 \mathrm{~min}$ in the experiments included in this article (preparations with higher spontaneous rates were not used). To avoid the large effects this spontaneous rhythm can have on evoked responses (Wu et al., 1990, 1991), stimuli that were scheduled to occur during or just after a spontaneous event were postponed for at least $1 \mathrm{~min}$. However, this precaution does not avoid the possibility that the siphon stimulus might trigger a spontaneous contraction. Nonetheless, such events could not have caused the response variability in the experiment using an intracellular electrode stimulus (Figs. 3,7 ) because there were no contractions in response to these stimuli. We do not know about the contribution that other possible intrinsic rhythms might make to the response variability but, clearly, systematic effects can be quite large.

We used an empirical definition of random and systematic variations. While it is possible, or even likely, that the random variations may have random origins, it is also possible that several independently cycling intrinsic rhythms could give rise to variability that would be empirically indistinguishable from apparently random variability. Symmetrically, apparently systematic variations could arise from the random activity of an interneuron with widespread effects.

\section{The activity of individual neurons is not a good predictor of the behavioral output}

The results presented in Figure 11 showed that, in general, the activity of individual neurons was only poorly correlated with the behavioral output. We have described two kinds of variability that would tend to reduce the correlations between individual neurons and the behavior. First, there is Poisson noise in the spike counts of individual neurons (Fig. 4). Second, even when there are systematic effects on the time of peak activity of the neuron population, the time of peak activity of individual neurons is not tightly locked to the population effect (Fig. 8, middle).

On the other hand, the summed activity of several groups, as defined by their response probabilities, was highly correlated with the behavioral output. This was true even for a group with a low $(0.13)$ response probability. We presume that other, equally arbitrary, groupings of neurons would also have a summed output that matched the behavior.

Even though the activity of individual neurons is not well correlated with the overall behavior, some neurons do have activity that is apparently correlated with the first phase of the gill withdrawal. Some of these neurons (e.g., 376-458 in Fig. 10) may be of the type Group I (Falk et al., 1993), which might be more closely related to the stimulus than to behavioral output.

\section{Animal-to-animal differences}

Comparison of the results of optical recordings from different preparations must be done cautiously. First, even though the stimulus strength used in the three trials illustrated in Figure 11 was the same, the location of the stimulus on the siphon could not be identical. Second, comparisons of recordings from different preparations need to take into account differences in the optical properties of the preparation. If the voltage-sensitive dye staining or the amount of scattering varies from animal to animal, then the completeness of the optical recording will also vary. In the absence of independent measurements of completeness, we cannot use the number of optically detected cells as an indication of the number of cells that were actually active. The differences in the number of cells in the raster diagrams of Figure $11(49,81$, and 61 neurons for animals 1,2 , and 3$)$ may be due either to real differences in the actual number of active neurons or to differences in the completeness of the optical recording.

It is unlikely that differences in the completeness or small differences in the position of the mechanical stimulus would affect the time course of the response. As Figures 10-12 show, there are substantial differences in time course from animal to animal, much larger than the differences found when comparing different trials in one animal. Similarly, there are substantial animal-to-animal differences in the distribution of spikes among neurons (Fig. 14). The raster diagrams from the 30 preparations also differ with regard to several additional properties (e.g., consistency in the timing of the peak response). These differences are large enough to suggest that with complete recordings it 
might be possible to distinguish every individual Aplysia simply by measuring the abdominal ganglion response to a light touch.

The very large animal-to-animal differences in synaptic strength at a single identifiable synapse reported by Gardner (1986) (mean synaptic conductance ranged from 0.028 to 1.7 $\mu \mathrm{S}$ ) did not necessarily imply large functional differences. It is possible that this synaptic function is carried out by several synapses acting in parallel and that the sum of their synaptic effects could be much more consistent than an individual synapse. Gardner (1990) did find that the range of synaptic strengths was reduced from a factor of 61 to a factor of 12 when the sum of synaptic strengths at four synapses in each animal was considered instead of a single synaptic strength. The number of neurons in 16 buccal hemiganglia from eight Navanax ranged from 149 to 288 (London et al., 1987), almost a factor of 2 . Similarly, large differences were found in the counts of neurons in Aplysia (Cash and Carew, 1989). In contrast, the number of neurons in nematodes (Caenorhabditis elegans) and the number in segmental ganglia of leeches (Hirudo medicinalis and Haementeria ghilianii) are much more consistent from animal to animal (Macagno, 1980; White et al., 1986). The number of neurons in $C$. elegans is said to be invariant from animal to animal. The means, SDs, and CVs for the five species are shown in Table 1. [A reviewer called our attention to the fact that the value for Aplysia comes from measurements of only two animals, and thus the CV for Aplysia is very uncertain. However, Coggeshall (1967) counted 1100 neurons in an abdominal ganglia from another small adult, a result that is consistent with a large CV for the number of neurons in Aplysia.]

Other characteristics of Aplysia also have relatively large CVs. Flinn et al. (1993) measured the 5-HT:dopamine ratio in the Aplysia nervous system at five different ages. The mean $\mathrm{CV}$ of the ratio was 0.24 . Similarly, Lloyd et al. (1985) found dramatic animal-to-animal differences in the number of small cardioactive peptide containing neurons in the Aplysia CNS.

The CVs of the number of neurons in the two opisthobranch ganglia are 25 times larger than those for the leech. However, the animal-to-animal differences in opisthobranchs might result from shifting of neurons among central ganglia or between central ganglia and the periphery, or from differing numbers of neurons serving identical functions. Thus, the functional meaning of differences in neuron numbers is not known.

The animal-to-animal differences in synaptic strength, neuron number, and transmitter content can be given explanations that would not imply large functional differences between animals. However, our finding, of substantial differences in neuronal response between animals, is consistent with the notion that the differences in synaptic strength and in number of neurons may have important functional consequences. Large animal-to-animal differences increase the difficulty in analyzing the neuronal circuits responsible for the behaviors generated by the ganglia.

As an example of individual-to-individual differences in a nervous system with many more neurons, Ojemann et al. (1989) mapped cortical sites where electrical stimulation induced naming errors in 117 humans. They found dramatic individual differences in the locations of these functional speech areas and concluded that "neither the location nor the absence of language function can be reliably predicted by anatomical considerations."

The animal-to-animal variability in synaptic strength, neuronal responsc, transmitter ratios, and cell numbers in Aplysia may have either genetic or environmental sources. Included in the possible environmental sources are short-term variables such as differences in circadian time or in time since the last feeding as well as longer-term and more difficult to control variables such as hormonal state or time of year.

There were, however, characteristics where animal-to-animal differences in Aplysia were difficult to detect. The relationship of the $\mathrm{CV}$ to the mean response and the broad distributions of the probability of correct discrimination and the response probability were all similar from animal to animal. Furthermore, comparison of the $\mathrm{CV}$ and its relationship to the mean response in Aplysia and in several mammalian species reveals that there are also similarities across phyla (Heggelund and Albus, 1978; Tolhurst et al., 1983).

\section{A distributed versus a discrete organization}

The neurons involved in the response to the mechanical touch have a broad distribution of probabilities of correct discrimination and response probabilities. There is a small fraction of the neurons that have a large probability of correct discrimination and a large response probability indicating that they might be important for the behavior. Another fraction of the neurons have a response probability of zero and a low probability of correct discrimination, suggesting that they are not involved in the response and may not helong to the same neuronal pool. However, the majority of neurons have scores between these extremes. We presume that a quantitative accounting for the behaviors generated by the abdominal ganglion would also require consideration of this large group of neurons.

One characteristic of a nervous system that functions in a distributed manner is that a large number of neurons are involved in each behavior. Several results presented in this article are consistent with this characteristic. First, the responses of individual neurons fluctuate from trial to trial. If a consistent behavioral output is to be produced, then the summed activity of groups of neurons must be integrated to generate the output. Second, the neuron population has a broad distribution of probability of correct discrimination and response probabilities. Finally, in somc preparations few, if any, individual ncurons have activity that is well correlated with the behavioral output.

Noise and inconsistency in the activity of individual neurons may be either detrimental or beneficial to the animal. It seems likely that noise would be detrimental in situations where high reliability is important or in situations where errors might have large consequences. On the other hand, during development noise might contribute to generating behavioral variation. The animal might then learn to associate these accidentally generated new behaviors with outcomes that could have positive survival value.

\section{References}

Byrne JH, Koester J (1978) Respiratory pumping: neuronal control of a centrally commanded behavior in Aplysia. Brain Res 143:87105 .

Byrne JH, Castellucci VF, Kandel ER (1978) Contributions of individual mechanoreceptor sensory neurons to defensive gill-withdrawal reflex in Aplysia. J Neurophysiol 41:418-431.

Cash D, Carew TJ (1989) A quantitative analysis of the development of the central nervous system in juvenile Aplysia californica. J Neurobiol 20:25-47.

Coggeshall RE (1967) A light and electron microscope study of the abdominal ganglion of Aplysia californica. J Neurophysiol 30:12631287.

Cohen TE, Henzi V, Kandel ER, Hawkins RD (1991) Further be- 
havioral and cellular studies of dishabituation and sensitization in Aplysia. Soc Neurosci Abstr 17:1302.

DeGroot MH (1986) Probability and statistics. Reading, MA: Addison-Wesley.

del Castillo J, Katz B (1954) Quantal components of the end-plate potential. J Physiol (Lond) 124:560-573.

Falk CX, Wu JY, Cohen LB, Tang C (1993) Non-uniform expression of habituation in the activity of distinct classes of neurons in the Aplysia abdominal ganglion. J Neurosci 13:4072-4081.

Flinn JM, Hong C, Holt RW, Chandhoke V (1993) Serotonin/dopamine ratio varies across the lifespan in Aplysia. Soc Neurosci Abstr 19:17

Frost WN, Clark GA, Kandel ER (1988) Parallel processing of shortterm memory for sensitization in Aplysia. J Neurobiol 19:297-334.

Gardner D (1986) Variations in amplitude and time course of inhibitory postsynaptic currents. J Neurophysiol 56:1424-1438.

Gardner D (1990) Paired individual and mean postsynaptic currents recorded in four cell networks in Aplysia. J Neurophysiol 63:12261240.

Green DM, Swets JA (1966) Signal detection theory and psychophysics. New York: Wiley.

Grinvald A, Hildesheim R, Gupta R, Cohen LB (1980) Better fluorescent probes for optical measurement of changes in membrane potential. Biol Bull 159:484.

Grinvald A, Cohen LB, Lesher S, Boyle MB (1981) Simultaneous optical monitoring of activity of many neurons in invertebrate ganglia using a 124-element photodiode array. J Neurophysiol 45:829-840.

Hawkins RD, Castellucci VF, Kandel ER (1981) Interneurons involved in mediation and modulation of the gill-withdrawal reflex in Aplysia. I. Identification and characterization. J Neurophysiol 45: 304-314.

Heggelund P, Albus K (1978) Response variability and orientation discrimination of single cells in striate cortex of the cat. Exp Brain Res 32:197-211.

Kanz JE, Eberly LB, Cobbs JS, Pinsker HM (1979) Ncuronal correlates of siphon withdrawal in freely moving Aplysia. $\mathbf{J}$ Neurophysiol 42 : $1538-1556$.

Koester J, Kandel ER (1977) Further identification of neurons in the abdominal ganglion of Aplysia using behavioral criteria. Brain Res 121:1-20.

Kuffler SW, Fitzhugh R, Barlow HB (1957) Maintained activity in the cat's retina in light and darkness. J Gen Physiol 40:683-702.

Kupfermann I, Pinsker H, Castellucci V, Kandel ER (1971) Central and peripheral control of gill movements in Aplysia. Science 174: $1252-1256$.

Lloyd PE, Mahon AC, Kupfermann I, Cohen JL, Scheller RH, Weiss KR (1985) Biochemical and immumocytological localization of molluscan small cardioactive peptides in the nervous system of $A p l y$ sia californica. J Neurosci 5:1851-1861.

London JA, Zecevic D, Cohen LB (1987) Simultaneous optical recording of activity from many neurons during feeding in Navanax. $\mathrm{J}$ Neurosci 7:649-661.
Macagno ER (1980) Number and distribution of neurons in leech segmental ganglia. J Comp Neurol 190:283-302.

Nakashima M, Yamada S, Shiono S, Maeda M, Sato F (1992) 448Detector optical recording system: development and application to Aplysia gill-withdrawal reflex. IEEE Trans Biomed Eng 39:26-36.

Ojemann G, Ojemann J, Letich E, Berger M (1989) Cortical language localization in left, dominant hemisphere: an electrical stimulation mapping investigation in 117 patients. J Neurosurg 71:316-326.

Salzberg BM, Grinvald A, Cohen LB, Davila HV, Ross WN (1977) Optical recording of neuronal activity in an invertebrate central nervous system: simultaneous monitoring of several neurons. J Neurophysiol 40:1281-1291.

Tang AC, Falk CX, Cohen LB, Wu JY (1992) The majority of neurons in the Aplysia abdominal ganglion have unreliable responses to siphon stimulation. Soc Neurosci Abstr 18:712.

Tolhurst DJ, Movshon JA, Dean AF (1983) The statistical reliability of signals in single neurons in cat and monkey visual cortex. Vision Res 23:775-785.

Trudeau LE, Castellucci VF (1992) Contribution of polysynaptic pathways in the mediation and plasticity of Aplysia gill and siphon withdrawal reflex: evidence for differential modulation. $J$ Neurosci 12 : $3838-3848$.

Tsau Y, Wu JY, Hopp HP, Cohen LB, Schiminovich D, Falk CX (1994) Distributed aspects of the response to siphon touch in Aplysia: spread of stimulus information and cross correlation analysis. $\mathbf{J}$ Neurosci, in press.

Werner G, Mountcastle VB (1963) The variability of central neural activity in a sensory system, and its implications for the central reflection of sensory events. J Neurophysiol 26:958-977.

White JG, Southgate E, Thomson JN, Brenner S (1986) The structure of the nervous system of the nematode Caenorhabditis elegans. Philos Trans R Soc Lond [Biol] 314:1-340.

Woolson RF (1987) Statistical methods for the analysis of biomedical data. New York: Wiley.

Wu JY, Cohen LB (1993) Fast multisitc optical mcasurements of membrane potential. In: Fluorescent and luminescent probes for biological activity (Mason WT, ed), pp 389-404. London: Academic.

Wu JY, Falk CX, Hopp HP, Cohen LB (1989) Trial-to-trial variability in the neuronal response to siphon touch in the Aplysia abdominal ganglion. Soc Neurosci Abstr 15:1264.

Wu JY, Roschin V, Cohen LB, Falk CX, Cohen AI (1990) Comparison of activity in the Aplysia abdominal ganglion during spontaneous and touch elicited gill withdrawals. Soc Neurosci Abstr 16:19.

Wu JY, Cohen LB, Falk CX (1991) Comparison of neuronal activity in the Aplysia abdominal ganglion during respiratory pumping and the gill-withdrawal reflex. Soc Neurosci Abstr 17:549.

Zecevic D, Wu JY, Cohen LB, London JA, Hopp HP, Falk CX (1989) Hundreds of neurons in the Aplysia abdominal ganglion are active during the gill-withdrawal reflex. J Ncurosci 9:3681-3689. 